\title{
Rückkehr zur Herkunft
}

\section{Autosoziobiografien erzählen von der Klassengesellschaft}

\section{Eva Blome}

Online publiziert: 24. November 2020

(C) Der/die Autor(en) 2020

Zusammenfassung Der Artikel setzt sich mit einem in jüngster Zeit vermehrt zu beobachtenden Narrativ auseinander: der Erzählung von der Herkunft aus einem deklassierten Milieu. Im Mittelpunkt stehen dabei autosoziobiografische Texte und Filme, die aus der Perspektive einer erworbenen sozialen Distanz den Anspruch verfolgen, individuelle Erfahrungen des Klassenwechsels mit einer Analyse aktueller gesellschaftlicher Problemlagen zu verbinden. Diese Darstellungen werden zum einen hinsichtlich ihrer hybriden Gattungszugehörigkeit und ihres kommunikativen Anspruchs befragt. Zum anderen werden ihre dominierenden Erzählweisen untersucht. Dabei zeigt sich, dass genealogisch-kollektivierende Konstellationen eine wichtige Rolle spielen: Erstens werden Klassenverhältnisse als Generationenverhältnisse narrativiert; zweitens wird ein die einzelne Autosoziobiografie sprengendes Erzählverfahren verwendet, durch das sich ein intertextueller Zusammenhang von aufeinander verweisenden Geschichten des Klassenwechsels konstituiert.

\footnotetext{
E. Blome $(\triangle)$

Institut für Germanistik, Fakultät für Geisteswissenschaften, SLM I, Universität Hamburg, Überseering 35, Postfach \#15, 22297 Hamburg, Deutschland

E-Mail: eva.blome@uni-hamburg.de
} 


\title{
Return to the origin
}

Autosociobiographies are reporting from the class society

\begin{abstract}
The article deals with a narrative that has received increasing attention in recent years: the story of one's social origin in a disadvantaged milieu. The focus is on autosociobiographical texts and films which, from the perspective of acquired social distance, aim at linking individual experiences of a change of social class with an analysis of current social problems. These representations are analyzed regarding their hybrid genre and their communicative ambitions. Furthermore, dominant narrative styles are examined. It turns out that configurations, which are both genealogical and collectivizing in kind, are of particular importance: First, relationships of class are narrated as generational relationships; Second, a narrative method is employed that transgresses the individual autosociobiography as it establishes intertextual connections among different stories of class change that refer to one another.
\end{abstract}

Von Möglichkeiten und Chancen, Begrenzungen und Risiken sozialer Mobilität zu erzählen hat Konjunktur. Dies erfolgt in letzter Zeit immer häufiger im narrativen Modus der Introspektion des Herkunftsmilieus aus der Perspektive so genannter Bildungsaufsteiger*innen. Die Beispiele sind ebenso zahlreich wie vielfältig: Journalistisch orientierte Erkundungen der eigenen Herkunft ${ }^{1}$ stehen neben soziologischen Essays und politischen Manifesten, die ihren Ausgang von der Autobiografie ihrer Autoren und deren sozialen und familiären Wurzeln nehmen. ${ }^{2}$ Britische und französische Filme wie Richard Billinghams Ray \& Liz (2019) oder Ladj Lys Les Misérables (2019), die zwischen Fiktion und Dokumentation changieren, skizzieren die sozialen Verwerfungen des Viertels der Kindheit oder ihrer Familien.

Vor allem aber haben international Texte Erfolg, die zugleich einen literarischen wie auch soziologischen Anspruch verfolgen und aus Sicht eines erwachsenen Erzählers von einer Auseinandersetzung mit dessen sozio-kultureller Herkunft berichten. Es handelt sich dabei um Darstellungen individueller Lebensgeschichten, die von einem Klassenwechsel qua Bildung und dessen Hindernissen erzählen und sich zugleich als Analysen der Mechanismen von Reproduktion und Nicht-Reproduktion bestehender Sozialverhältnisse zu erkennen geben. Wichtige literarische Impulse kommen aus Frankreich: Didier Eribons Retour à Reims (2009) war sicherlich das einflussreichste und meist diskutierte Buch, das in diesem Zusammenhang genannt werden muss. Saša Stanišićs preisgekröntes Buch mit dem prägnanten Titel Herkunft kann stellvertretend als deutschsprachiger Bestseller des Jahres 2019, Tara Westovers Educated (2018) als US-amerikanischer und Darren McGarveys Poverty Safari (2017) als britischer Erfolgstitel angeführt werden. Mit Bov Bjergs Serpentinen (2020) und Christian Barons Ein Mann seiner Klasse (2020) sind vor nicht allzu

\footnotetext{
1 Marco Maurer, Du bleibst was du bist. Warum bei uns immer noch die soziale Herkunft entscheidet, München 2015; Patrick Bauer, Die Parallelklasse. Ahmed, ich und die anderen - Die Lüge von der Chancengleichheit, München 2011.

2 Steffen Mau, Lütten Klein. Leben in der ostdeutschen Transformationsgesellschaft, Berlin 2019; Paul Collier, Sozialer Kapitalismus. Mein Manifest gegen den Zerfall unserer Gesellschaft, München 2019.
} 
langer Zeit zwei weitere Titel deutscher Autoren erschienen, die diesem Narrativ folgen.

Offenbar stoßen diese Erzählformen in Zeiten, in denen die Segregation von Arm und Reich immer deutlicher zu Tage tritt und nicht zuletzt auch die Schulen wohlhabender und benachteiligter Stadtteile immer unterschiedlichere Aufgaben zu bewältigen haben, ${ }^{3}$ in Öffentlichkeit und Wissenschaft auf große Resonanz. Sie werden als Seismografen für eine Situation betrachtet, in der nationale wie internationale Vergleichsstudien die Reproduktion sozialer Ungleichheit durch das Bildungssystem immer wieder nachdrücklich herausstellen. In Deutschland, so haben die Ergebnisse der letzten PISA-Studie erneut belegt, hängt der Bildungserfolg in besonders hohem Maß von der sozialen Herkunft ab; zugleich glaubt ein Großteil der Deutschen weiterhin an die Möglichkeit eines sozialen Aufstiegs durch eigene Bildungsbemühungen. ${ }^{4}$ Vor allem aber werden diese Texte im Zusammenhang mit dem Erstarken des Rechtspopulismus als aussagekräftige Zeugnisse herangezogen, die helfen sollen, dessen Ursachen zu erklären. Sie erscheinen, auch aus ethnologischer und erziehungswissenschaftlicher Perspektive, schließlich deshalb als so interessant, weil sie als Möglichkeit verstanden und rezipiert werden, aus der Sicht von >doppelten Insidern< intime Einblicke in die sich vermeintlich verstärkende Kluft zwischen akademischen und nichtakademischen Sozialkulturen sowie zwischen »Klassenaufsteigern und den etablierten linksliberalen Milieus $\ll^{5}$ zu gewähren.

Die Rede von einem »Bildungsaufstieg « ist in diesem Zusammenhang nicht unproblematisch: Reproduziert der Begriff doch ein - zumeist implizites - hegemoniales Verständnis von Bildung ebenso wie die Vorstellung einer Gesellschaftsstruktur,

\footnotetext{
3 Vgl. Marcel Helbig, Stefanie Jähnen, »Wie brüchig ist die soziale Architektur unserer Städte? Trends und Analysen der Segregation in 74 deutschen Städten, Discussion Paper«; in: https://www.econstor.eu/ bitstream/10419/179001/1/p18-001.pdf (07.02.2020).

4 Vgl. Elisabeth Grewenig et al., »Was die Deutschen über Bildungsungleichheit denken. Ergebnisse des ifo Bildungsbarometers 2019«, ifo Schnelldienst 72/17 (2019), 3-17; abrufbar unter https://www.ifo.de/ DocDL/sd-2019-17-woessmann-etal-bildungsbarometer-2019-09-12.pdf (07.02.2020).

5 Jens Wietschorke, »What's the Matter with Fergus Falls? Reisen ins Herz der politischen Rechten«, Merkur 844 (Sept. 2019), 76-84, hier: 83; vgl. auch Markus Rieger-Ladich, »Cooling down. Warum Bildung mehr ausgrenzt als inkludiert«, Kursbuch 193 (März 2018) (Schwerpunkt: »301 Gramm Bildung«), 100114.

Nicht nur Ethnologen (Wietschorke) und Erziehungswissenschaftler (Rieger-Ladich), sondern auch soziologische Studien der jüngeren Vergangenheit nehmen literarische Texte bezeichnenderweise zum Ausgangspunkt ihrer Gegenwartsdiagnosen, so etwa Oliver Nachtwey in Die Abstiegsgesellschaft. Über das Aufbegehren in der regressiven Moderne (Berlin 2016, 8 f.). Darin werden gleich zu Beginn Texte wie Ulla Hahns Selbstlebensbeschreibung in mehreren Romanbänden beginnend mit Das verborgene Wort (2001), Robert Kischs Möbelhaus (2015), Thomas Melles 3000 Euro (2014), Katharina Hackers Die Habenichtse (2006), Silke Scheuermanns Die Häuser der anderen (2012) und einige mehr als sensible Seismografen für einen Wandel angeführt, deren Beschreibung sich Nachtwey widmet, indem er die These verfolgt, dass »[a]us der Gesellschaft des Aufstiegs und der sozialen Integration [...] eine Gesellschaft des sozialen Abstiegs, der Prekarität und Polarisierung geworden« (8) sei. Zum Narrativ des sozialen Abstiegs vgl. aus literaturwissenschaftlicher Perspektive auch Markus Steinmayr, »Abstieg trotz Bildung. Inszenierungen sozialer Unsicherheit in der Gegenwartsliteratur (Melle, Kisch, Bilkau)«, Internationales Archiv für Sozialgeschichte der Deutschen Literatur 44 (2019), 100-131.
} 
die zwischen »oben« und »unten « unterscheidet. ${ }^{6}$ Auch klingt die Erzählung vom Bildungsaufstieg zunächst nach einer Erfolgsgeschichte; mithin verdeckt der Begriff womöglich die individuellen Kosten und Probleme, die mit einem solchen Lebensweg verbunden sind. ${ }^{7}$ Die Vorstellung eines Bildungsaufstiegs und erst recht die Bezeichnung von Individuen als Bildungsaufsteiger*innen gilt daher oftmals als fragwürdig, wird dadurch doch immer auch die Annahme einer (ursprünglichen) Bildungsferne mittransportiert. ${ }^{8}$ Im Gegensatz zu möglichen alternativen Konzepten wie »Klassenwechsel« oder »Klassenübergang « hat die Idee eines Bildungsaufstiegs jedoch den Vorteil, den Umstand sichtbar zu machen, dass Klassenverhältnisse und Bildungsverhältnisse in einer engen Abhängigkeitsbeziehung stehen, die sich individuellen Lebenswegen einschreibt - wenngleich auf je sehr unterschiedliche Art und Weise. Entsprechend wird dem Narrativ von der Herkunft aus einem deklassierten Milieu dann eine besondere gesellschaftsanalytische Kraft zugeschrieben, wenn es vom Standpunkt einer aufgrund von Bildung erworbenen, mit einer spezifischen Entfremdungserfahrung einhergehenden sozialen Distanz ausgestattet ist.

Die folgenden Ausführungen stellen also Erzählungen in den Mittelpunkt, die von einem mithilfe von Bildung erworbenen Klassenwechsel sowie den mit ihm verbundenen Chancen und Risiken berichten und zugleich Beobachtungen allgemeiner Art zu aktuellen Phänomenen gesellschaftlicher (Nicht-)Mobilität anstellen. Ihr Ziel ist es, anhand eines Korpus neuerer Texte und Filme das diesen Erzählungen zugrunde liegende narrative Muster sichtbar zu machen. ${ }^{9}$ Dafür werden sie zunächst in gattungstypologischer Hinsicht im Kontext autosoziobiografischer Dar-

\footnotetext{
${ }^{6}$ Dass der Begriff des Bildungsaufstiegs nicht ohne Weiteres ins Englische oder Französische übersetzt werden kann, wo Formulierungen wie »educational upward mobility « oder - in einer Formulierung von Chantal Jaquet - »réussite scolaire et [...] ascension sociale« (zit. nach Chantal Jaquet, Les transclasses ou la non-reproduction, Paris 2014, 4) benutzt werden, hängt wohl auch mit dem Konzept der Bildung als eines genuin »deutschen Deutungsmusters« (Georg Bollenbeck) zusammen. (Vgl. dazu Eva Blome, Patrick Eiden-Offe, Manfred Weinberg, »Klassen-Bildung. Ein Problemaufriss«, Internationales Archiv für Sozialgeschichte der deutschen Literatur 35/2 [2010], 158-194, insbesondere 173f.) Für die Frage nach der Übersetzbarkeit des Begriffs des Bildungsaufstiegs sowie für weitere Hinweise zum vorliegenden Artikel danke ich Nacim Ghanbari.

7 Vgl. dazu die Überlegung Marcus Steinmayrs: »Für die Literaturwissenschaft gilt es somit, in das sozialpolitische beziehungsweise sozioökonomische Einvernehmen über die positiven Eigenschaften des Bildungsaufstiegs zu intervenieren. Literatur, das literarische Schreiben wäre dann die Bilanzierung jener individuellen und sozialen Kosten des Bildungsaufstiegs.« (Steinmayr [Anm. 5], 131.).

8 Vgl. Roland Reichenbach, »Über Bildungsferne«, Merkur 795 (Aug. 2015), 5-15. (Bezeichnend sind die »Autobiografische[n] Notizen«, mit denen Reichenbach seinen Essay beginnt.).

9 Die hier analysierten Beispiele stammen überwiegend aus dem französischen und deutschsprachigen, aber auch britischen Kontext. Dass sich autosoziobiografische Erzählverfahren und das Narrativ der Rückkehr zur sozial-familiären Herkunft trotz sehr unterschiedlicher nationaler bildungsinstitutioneller und sozialgeschichtlicher Voraussetzungen in Großbritannien, Frankreich und Deutschland in den letzten Jahrzehnten in nicht komplett gleicher, aber doch vergleichbarer Weise verbreitet haben, bedürfte einer eigenen genaueren Erörterung. Diese hätte Prozesse narrativer und formaler Rezeption und Adaption ebenso wie diejenigen der Variation und Transformation zu berücksichtigen. Autosoziobiografien könnten so über die genannten (national-)sprachlichen Kontexte hinaus - als mögliches Beispiel für »traveling genres« (Margaret Cohen) in den Blick genommen werden. Die folgenden Ausführungen fokussieren nicht auf diese Fragestellung, mögen aber vielleicht zu deren Vertiefung in weiteren Untersuchungen anregen - dies vor allem insofern der Aspekt der öffentlichen und literarischen Rezeption französischer Autosoziobiografien und Theoriekontexte durch anders- und insbesondere deutschsprachige Texte und Diskurse im vorliegenden Aufsatz durchaus zentral ist. Außerdem greift die hier geleistete Auseinandersetzung mit
} 
stellungsverfahren verortet (Abschnitt I), um sodann die spezifischen Erzählweisen, die das Narrativ einer Rückkehr zur sozialen Herkunft mit besonderer Evidenz ausstatten, einer genaueren Analyse zu unterziehen. Aufgezeigt wird, dass für die autosoziobiografische Narration des Klassenübergangs genealogisch-kollektivierende Konstellationen von besonderer Bedeutung sind - und zwar in doppelter Hinsicht: Zum einen werden Klassenverhältnisse als Generationenverhältnisse erzählt (Abschnitt II). Dabei kommt der narrativen Einbindung von Fotografien auf der Ebene der histoire eine zentrale Rolle zu (Abschnitt III), während hinsichtlich des discours Fragen der Repräsentation und der Adressierung bestimmend sind (Abschnitt IV). Zum anderen führen Verfahren der Intertextualität zu einer trans- und intragenerativ ausgerichteten Herstellung eines Textzusammenhangs verschiedenster, aufeinander verweisender Geschichten des Klassenwechsels (Abschnitt V). Dass diese sowohl literatur- als auch sozialwissenschaftliches Interesse auf sich ziehen, ${ }^{10} \mathrm{kann}$ schließlich als Ausgangspunkt dienen, um neue Fragen zum Verhältnis von Ästhetik und Gesellschaft, Erzählforschung und Soziologie aufzuwerfen (Abschnitt VI).

\section{I.}

\section{AUTOSOZIOBIOGRAFISCHES ERZÄHLEN}

Die im Folgenden untersuchten Texte und Filme zeichnen sich zunächst einmal durch die Gemeinsamkeit aus, dass sie alle - wenngleich auf je sehr unterschiedliche Weise - zwischen Autobiografie, Fiktion und Gesellschaftsanalyse oszillieren und diese verschiedenen Dimensionen zu einem eigenen narrativen Format verbinden. ${ }^{11}$ Die individuelle Rückschau auf das Umfeld der sozialen Herkunft - oftmals die Arbeiterklasse oder ein bäuerliches Milieu - stellt dabei, so der mehr oder weniger explizit ausgestellte Anspruch, nicht nur eine Auseinandersetzung mit der Erfahrung des Klassenwechsels dar. Die Beschäftigung mit dem eigenen Lebensweg als einer Aufstiegsgeschichte und den - zumeist als unwahrscheinlich oder zufällig markierten - Bedingungen ihrer Möglichkeit ist vielmehr stets auch mit der Ambition verbunden, aus dieser Perspektive zugleich einen soziologisch-literarischen

autosoziobiografischen Erzählweisen bis in die 1970er Jahre aus und kann so womöglich zu einer größeren historischen Tiefenschärfe der Diskussion autosoziobiografischer Texte beitragen.

10 Als eines der jüngsten Beispiele zur soziologischen Perspektivierung von Narrativen des Klassenwechsels vgl. Julia Reuter, »Literarische Selbstzeugnisse von Bildungsaufsteiger*innen zwischen Autobiografie und Sozioanalyse«, in: Julia Reuter, Markus Gamper, Christina Möller, Frerk Blome (Hrsg.), Vom Arbeiterkind zur Professur. Sozialer Aufstieg in der Wissenschaft. Autobiographische Notizen und soziobiographische Analysen, Bielefeld 2020, 103-128. (Diese Veröffentlichung lag mir erst nach Abschluss der Arbeit an diesem Artikel vor und konnte daher in den folgenden Ausführungen leider nicht weiter berücksichtigt werden.).

11 Die zu konstatierenden Unterschiede liegen dabei insbesondere darin, wie sich das Verhältnis von Dokumentation und Literarizität, Fiktion und Faktizität in jedem Einzelfall einer autosoziobiografischen Darstellung ausweisen lässt und wie sehr der soziologische Anspruch der einzelnen Texte oder Filme von diesen selbst expliziert wird. Damit zusammenhängend ist die Frage der Genrezuordnung der Autosoziobiografien von besonderem Interesse, die nach einer hermeneutischen Beantwortung verlangt. Im Folgenden werden dazu mit Blick auf einzelne Beispiele autosoziobiografischen Erzählens einige Hinweise gegeben, jedoch bedarf die Frage nach Gattung und Autosoziobiografie noch einer dezidierteren Ausarbeitung. 
Blick auf gesellschaftspolitisch relevante Entwicklungen der Gegenwart zu werfen. Die (literarische) Erzählung, die ihren Ausgang vom Individuum nimmt, ermöglicht dabei eine besondere Sicht auf Phänomene sozialer (Nicht-)Mobilität, weil sie eine Unmittelbarkeit des Zugangs sowie eine Dichte und Authentizität des Berichts ermöglicht, wie sie auf anderem Weg, etwa durch empirische Untersuchungen und Statistiken, nicht zu erlangen ist. Annie Ernaux prägte die inzwischen recht geläufige Bezeichnung eines solchen Erzählens als autosoziobiografisch (»auto-sociobiographiques «). ${ }^{12}$

Für die Einordnung dieser Textgattung erscheint in einem ersten Schritt der Hinweis auf Ähnlichkeiten mit autobiografischen und auch autofiktionalen Darstellungen sinnvoll. Das zentrale Merkmal autobiografischer Erzählverfahren, das nach Philippe Lejeune darin besteht, dass der Text eine Identität von Autor, Erzähler und Hauptfigur nahelegt, trifft auf die hier diskutierten Beispiele zu. ${ }^{13}$ Dieser >autobiografische Pakt< stellt, so lässt sich jedoch feststellen, für das autosoziobiografische Erzählen lediglich ein notwendiges, aber kein hinreichendes Kriterium dar. An der derzeit zu beobachtenden Konjunktur autofiktionaler Darstellungen haben autosoziobiografische Texte wiederum insofern Anteil, als dass der »Glaube an die Realität ${ }^{14}$ für beide Genres zentral ist. Sie gehen aber nicht ineinander auf. Denn während aktuelle Autofiktionen ihre Aufmerksamkeit zwar ebenfalls auf das eigene Leben richten, oftmals auch auf Alltäglichkeiten und kleinste Details, spielen in ihnen Fragen der sozialen und familiären Genealogie zumeist nur eine untergeordnete Rolle. ${ }^{15}$ Zudem beansprucht nicht jede autobiografisch grundierte Erkundung der familiären und sozialen Herkunft, zugleich eine, und sei es auch nur implizite, Analyse gesellschaftlicher Problemlagen zu sein. ${ }^{16}$ Für Andreas Maiers auf elf Bände ange-

12 Annie Ernaux, L'écriture comme un couteau. Entretien avec Pierre-Yves Jeannet, Paris 2011 [2003], 23.

13 Vgl. Philippe Lejeune, Le pacte autobiographique, Paris 1975 (dt.: Philippe Lejeune, Der autobiographische Pakt, Frankfurt a.M. 1994).

14 Claude Haas, »Vom Untergang der Autobiografie im Strudel der Autofiktion. Oder: Realität heute«, Texte zur Kunst 115 (Sept. 2019), 78-91, hier: 87. Zur soziokulturellen Einordnung autofiktionalen Erzählens vgl. Toni Tholen, »Facetten autofiktionalen Schreibens seit den 1970er Jahren - die Automelanchographie «, in: Toni Tholen, Patricia Cifre Wibrow, Arno Gimber (Hrsg.), Fakt, Fiktionen und Fact-Fictions, Hildesheim 2018, 39-57.

15 Als naheliegendes Beispiel für die aktuelle Konjunktur eines selbstbezogenen autofiktionalen Erzählens kann hier Karl Ove Knausgårds exzessives, aus sechs Bänden bestehendes Selbstbeschreibungsprojekt Min Kamp (2009ff.) angeführt werden (vgl. dazu auch Haas [Anm. 14]).

16 Umgekehrt ist aber aktuell auch eine Konjunktur von Texten zu beobachten, die sich zwar nicht explizit als autobiografisch grundiert ausweisen, aber dennoch das Narrativ von der Rückkehr zur sozialen Herkunft aus der Perspektive von (gescheiterten) Bildungsaufsteiger*innen zum Thema haben. Dies gilt etwa für Bov Bjergs Serpentinen (2020) und Elena Ferrantes zwischen 2011 und 2014 auf Italienisch erschienene Neapolitanische Saga, für die bereits Julika Griem festgestellt hat, dass sie »sich wie eine weibliche Variation von [...] Rückkehr nach Reims « lese (Julika Griem, »Nahkampf auf der Langstrecke. Elena Ferrante und Karl Ove Knausgård «, Merkur 810 [November 2016], 62-69, hier: 67). Zudem kann in diesem Zusammenhang der Roman Faux départ von Marion Messina (2017) genannt werden, in dem die Hoffnungen der jungen, aus dem provinziellen Grenoble stammenden Heldin auf einen sozialen (Bildungs-)Aufstieg in Paris grandios und in aller Härte scheitern, weshalb die Rückkehr zu ihrer Herkunftsfamilie am Ende des Romans unausweichlich erscheint: »Ihre Eltern wussten, dass sie zurückkommen würde, und hatten keine Fragen gestellt.« (Marion Messina, Fehlstart, München 2020, 166.).

Anhand von Angelika Klüssendorfs Romantrilogie Das Mädchen (2011), April (2014) und Jahre später

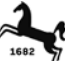


legtes literarisches Großprojekt Ortsumgehung (2010ff.), das sich der literarisierten Darstellung von Kindheit und Jugend in der Wetterau und den weiteren Irrungen, Wirrungen des Lebenswegs des Autors widmet, sowie für seine Sammlung von Kolumnentexten unter dem Titel Was wir waren (2018) trifft das zum Beispiel nicht zu - beharrt Maiers literarisches Alter Ego doch darauf, »ein Einzelwesen zu sein, das nicht kollektiviert werden kann und darf in gesellschaftlichen Utopien welcher Couleur auch immer $\ll .{ }^{17}$

Für ein besseres Verständnis des autosoziobiografischen Genres ist mehr zu gewinnen, wenn es in den Kontext von Rückkehrnarrativen eingeordnet wird. Das Sujet der Heimkehr wird in der Literaturgeschichte von der Antike bis zur Moderne wie auch in der literaturwissenschaftlichen Forschung zumeist als Rückkehr aus der geografisch, landschaftlich wie kulturell als alteritär gekennzeichneten Fremde gedacht und konzipiert. Innerhalb der literarischen Texte wird dabei üblicherweise die Frage verhandelt, ob die soziale Reintegration des Heimkehrers gelingt oder nicht. Über Erfolg oder Misserfolg entscheidet dabei nicht zuletzt der Umstand, ob der aus der fernen Fremde Rückkehrende in der Lage ist, sich als Erzähler seiner in den anderen Welten erlebten und erlittenen Abenteuer zu erweisen. ${ }^{18}$

Das Narrativ von der Rückkehr aus der sozialen Ferne und der Wiederannäherung an das Herkunftsmilieu unterscheidet sich hiervon in auffälliger Weise. Zwar macht es zuweilen bei dieser Tradition Anleihen, geht doch die Erzählung von einer sozialen Distanzierung der Bildungsaufsteiger*innen ebenfalls fast immer mit der Darstellung einer räumlichen Trennung einher (insbesondere nach dem Muster Stadt versus Land, Metropole versus Peripherie). Der große Unterschied ist jedoch in dem Umstand zu sehen, dass die Rückkehr in Autosoziobiografien nicht einmal potenziell als eine Rückkehr auf Dauer denkbar ist: Der soziale Rückkehrer bleibt vielmehr ein Wanderer zwischen den Welten respektive Klassen. Zudem ist die kommunikative Herausforderung eine völlig andere: Die Klassenwechsler*innen richten sich mit ihren Erzählungen üblicherweise nicht an die >Daheimgebliebenen $<$, sondern adressieren das soziale Ankunftsmilieu, also ein tendenziell akademisch gebildetes Lesepublikum. Die narrative Rückkehr dient folglich der Aufklärung der >anderen<, inzwischen mehr oder weniger zur eigenen gemachten sozialen Bezugsgruppe, dessen Vertreter*innen größtenteils nicht über die biografische Erfahrung eines Klassenwechsels verfügen.

Für die einzelne autosoziobiografische Darstellung kann daher gefragt werden, an welche Leserschaft genau sich diese jeweils richtet. Dabei ist auch der Fall möglich, dass ein über höhere Bildungstitel verfügendes Zielpublikum explizit nicht oder zu-

(2018) oder von Anke Stellings Schäfchen im Trockenen (2018) sowie von Zadie Smiths Swingtime (2016) oder Chimamanda Ngozi Adichies Americanah (2013) könnte des Weiteren diskutiert werden, ob überhaupt und ggf. inwiefern Grenzen zwischen dem aktuellen Gesellschaftsroman, der in einer spezifischen Variante der Gattung eben auch Herkunftsgeschichten involviert und/oder diese zum (autobiografisch grundierten) Ausgangspunkt seines Erzählens macht, und dem Genre der Autosoziobiografien gezogen werden können.

17 Andreas Maier, Was wir waren, Berlin 2018, Klappentext, o.S.

18 Vgl. Eva Eßlinger, »Vorwort«, in: Dies. (Hrsg.), »Nostos und Gewalt. Heimkehr in der Prosa des 19. und 20. Jahrhunderts « (=Themenschwerpunkt der Deutschen Vierteljahrsschrift für Literaturwissenschaft und Geistesgeschichte 92 [2018]), 119-126. 
mindest nicht in erster Linie adressiert wird. ${ }^{19}$ Für autosoziobiografische Texte stellt sich zudem das grundsätzliche Problem der Repräsentation der Herkunftskultur, die der Erzählerin oder dem Erzähler vertraut ist, inzwischen aber auch als fremd und alteritär erscheint. ${ }^{20}$

Doch wie funktioniert nun eigentlich die narrative Verknüpfung der Erzählung individueller Lebenswege mit dem Anspruch der Gesellschaftsanalyse? Und wie ist diese zu bewerten? Carlos Spoerhase hat angesichts der Konjunktur autosoziobiografischer Darstellungen auf die »Gefahr« hingewiesen, dass mit der Perspektivierung der Gesellschaft aus dem Blickwinkel des individuellen Aufstiegs »ein politischer Individualismus gestützt « werde, »der kollektive Problemlagen und Lösungsansätze verstellt «. ${ }^{21} »$ Literarische Formprobleme«, so Spoerhase, »können politische Folgeprobleme zeitigen: Das individuelle Schicksal darf nicht deshalb, weil es sich am besten erzählen läßt, zur maßgeblichen Leitgröße des politischen Denkens werden. «22 Besteht eine solche Gefahr tatsächlich? Und welche Umgangsweisen finden die autobiografischen Darstellungen selbst, um ihr gegebenenfalls zu begegnen?

Für eine Beantwortung dieser Fragen ist es zunächst hilfreich, das Verhältnis von Individualität und Kollektivität innerhalb autosoziobiografischer Darstellungen genauer zu bestimmen. Zwei Aspekte sind in diesem Zusammenhang herauszustellen: Erstens die Beobachtung, dass die hier diskutierten Beispiele Klassenverhältnisse als Verwandtschafts- und Generationenverhältnisse erzählen. Die Auseinandersetzung mit den Eltern und/oder Großeltern, oft ausgelöst durch den Tod eines Elternoder Großelternteils, wird zur Auseinandersetzung mit der Frage, welche Faktoren eine (Nicht-)Veränderung individueller sozialer Positionen in der Gesellschaft bedingen können. Bildung erscheint dabei zwar nicht als alleinige Einflussgröße, aber als eine entscheidende. Autosoziobiografien sind daher gerade nicht nur als Erzählungen von Individualität bzw. individueller Lebenswege aufzufassen. In einer gattungstheoretischen Perspektive ist vielmehr deren Nähe zum Familien- und Generationenroman in den Blick zu nehmen. ${ }^{23}$ Zweitens fällt ein Erzählverfahren auf,

\footnotetext{
19 So etwa Darren McGarvey, der mit seinem Buch Poverty Safari (2017) »vor allem Menschen ansprechen [möchte], die sich missverstanden und ungehört fühlen. [...] Ich habe versucht, für Leute wie mich zu schreiben, die Schwierigkeiten mit dem Lesen haben [...].«(Darren McGarvey, Armutssafari. Von der Wut der abgehängten Unterschicht, München 2019, 13 und 21.).

Vgl. im Gegensatz dazu Didier Eribons Aussage in Rückkehr nach Reims: »[...] natürlich weiß ich, dass die von mir so Beschriebenen wahrscheinlich nicht zu meinen Lesern gehören werden.« (Didier Eribon, Rückkehr nach Reims, Berlin 2016, 90.).

Ladj Ly stellt für seinen Film Les Misérables eine doppelte Adressierung und Wirkungsabsicht heraus: »Die einen sollen sehen, dass man es eben sehr wohl schaffen kann, in die elitären Kreise vorzudringen, während die anderen hoffentlich realisieren, dass sie sich keinen Gefallen tun, alle abzuweisen, die einen anderen Hintergrund haben. (»Ich wollte genau sein und gerecht«, Interview mit Patrick Heidmann, 22. Januar 2020, in: https://taz.de/Die-Wuetenden-Regisseur-ueber-Banlieues/!5655823/ (09.02.2020).).

${ }^{20}$ Zum Problem der Repräsentation und zu Fragen der Adressierung vgl. Abschnitt IV.

${ }^{21}$ Carlos Spoerhase, »Politik der Form. Autosoziobiografie als Gesellschaftsanalyse«, Merkur 818 (Juli 2017), 27-37, hier: 37.

${ }^{22}$ Carlos Spoerhase, »Aufstiegsangst: Zur Autosoziobiographie des Klassenübergängers«, in: Chantal Jaquet, Zwischen den Klassen. Über die Nicht-Reproduktion sozialer Macht, Konstanz 2018, 231-253, hier: 249.

${ }^{23}$ Im Gegensatz dazu beobachtet Spoerhase einen Ausfall des Generationen- und Familienromans im Kontext autosoziobiografischen Schreibens (vgl. Spoerhase [Anm. 22], 251).
} 
das den Rahmen der einzelnen Autosoziobiografie überschreitet, weil es auf einem intertextuellen Zusammenhang verschiedenster, aufeinander verweisender Geschichten des Klassenwechsels beruht. Daher gilt es, die Bedeutung eines Textumfelds zu erfassen, das der Schaffung einer Gemeinschaft einander ähnlicher Lebenswege von Klassenübergänger*innen dient.

\section{II.}

\section{KLASSENVERHÄLTNISSE ALS GENERATIONENVERHÄLTNISSE ERZÄHLEN}

Das in den letzten Jahren im Feuilleton und in der Wissenschaft - gerade auch in Deutschland - am intensivsten diskutierte und auch literarisch einflussreichste Beispiel autosoziobiografischen Schreibens ist Didier Eribons Buch Rückkehr nach Reims (2016, frz. Retour à Reims, 2009). In dem, vom Autor selbst auch als »nonfiktionaler Roman $\ll^{24}$ bezeichneten, Text kehrt der schwule Intellektuelle und Soziologe Eribon in das Arbeitermilieu seiner Familie im Nordosten Frankreichs zurück, um sich mit seiner $»$ Klassenflucht $\ll^{25}$ und $»$ Herkunftsscham $\ll{ }^{26}$ auseinanderzusetzen sowie am eigenen Beispiel die Mechanismen der gesellschaftlichen Reproduktion von Sozial- und Bildungsverhältnissen aufzuklären. ${ }^{27}$ Dabei ist der Tod von Eribons Vater Anlass und Bedingung dafür, sich der Herkunftsfamilie wieder zuzuwenden. ${ }^{28}$ Das Wiedersehen mit der verwitweten Mutter und die Auseinandersetzung mit ihrem Leben im Kontrast zum eigenen verknüpfen in Rückkehr nach Reims Herkunft und Gegenwart. Bildung und sexuelle Orientierung trennen den erwachsenen Ich-Erzähler von seiner Mutter - und beides hängt miteinander zusammen: Die Homophobie von Familienangehörigen und Herkunftsmilieu sind Antrieb einer Sehnsucht nach einer anderen sozialen Umgebung; Bildung ermöglicht den Fortgang in die weniger heteronormative Großstadt Paris.

Indes bleibt die Figur des verstorbenen Vaters in Rückkehr nach Reims randständig, mehr noch, sie bildet eine markante Leerstelle. Dass dies kein Zufall ist, wird in Eribons 2013 publizierten und seit 2018 auf Deutsch vorliegendem Buch Gesellschaft als Urteil deutlich. Hier bekennt der Autor gleich zu Beginn, dass er aus einer Fotografie, die auf dem Cover der deutschen Taschenbuchausgabe von Rückkehr nach Reims abgebildet ist, seinen Vater herausgeschnitten habe. ${ }^{29}$ Dieser Akt zeigt für Eribon die Ambivalenz und das Krisenhafte auf, das mit der Rückschau auf seine

24 »Didier Eribon zur Krise der Linken: >Ihr seid nicht das Volk««, Interview mit Jan Feddersen \& Rainer Nicolaysen, 23. September 2016, in: http://www.taz.de/!5340042/ (09.02.2020).

25 Didier Eribon, Gesellschaft als Urteil. Klassen, Identitäten, Wege, Berlin 2017, 106.

26 Eribon (Anm. 19), 19.

27 Zur Narration sozialer Ungleichheiten in Eribons Rückkehr nach Reims vgl. auch Eva Blome, »Institutionalisierung und Innovation. Gender Studies in literatur- und sozialwissenschaftlicher Perspektive (mit Überlegungen zu Didier Eribons Rückkehr nach Reims)«, in: Manuela Günter, Annette Keck (Hrsg.), Kulturwissenschaftliche Perspektiven der Gender Studies, Berlin 2018, 37-62, insb. 51-60.

28 »Sobald er weg war, wurde es mir möglich, diese Reise, oder besser, diesen Prozess der Rückkehr auf mich zu nehmen, zu dem ich mich so lange nicht hatte entschließen können.« (Eribon [Anm. 19], 10.).

29 Vgl. Eribon (Anm. 25), 17-25. 
soziale Herkunft und deren Darstellung verbunden ist: »Zeigen, was man geworden ist, ist angenehm und aufwertend. Zeigen, was man einmal war, ist es weniger. $\ll^{30}$ Die Eltern- und Großelterngeneration stehen für genau das, was man einmal war und nun nicht mehr ist, aber immer noch in sich trägt.

Dass der Tod oder ein sonstiger Verlust von Eltern oder Großeltern als Anlass autosoziobiografischen Erzählens dient und von den Texten als ein solcher hervorgehoben wird, erscheint als spezifisches Strukturmerkmal autosoziobiografischer Darstellungen. ${ }^{31}$ Die Auseinandersetzung mit der familiären Genealogie rückt die Texte in die Nähe von Familienchroniken. Und da »Aufstiegsgeschichten [...] auch immer Bildungsgeschichten « ${ }^{32}$ sind, weisen sie zudem Ähnlichkeiten zum Bildungsroman auf, nehmen dabei jedoch eine >umgekehrte< Form an: Sie gehen von einer bereits vollzogenen Entwicklung aus und erkunden deren individuellen, familiären und sozialen Voraussetzungen. Diese begegnen in Form von Erinnerungen an das Vergangene und schließen an den Verlust von Familienangehörigen an. So formuliert etwa Stanišić in seinem Buch Herkunft, das seinen Ausgangspunkt von der zunehmenden Demenz der Großmutter des Erzählers nimmt: »Als meine Großmutter Kristina Erinnerungen $\mathrm{zu}$ verlieren begann, begann ich, Erinnerungen $\mathrm{zu}$ sammeln. $\ll^{33}$ Aber auch deutlich frühere Texte wie Ernaux' La Place (1984) und Une femme (1987) haben bereits die Form von Erinnerungsbüchern: La Place widmet sich dem Leben des Vaters, Une femme dem Leben der Mutter, in beiden Fällen löst der Tod des Elternteils Erinnern und Erzählen aus. ${ }^{34}$ Wenn Eribon in Auseinandersetzung mit Texten Ernaux' aus den 1980er Jahren den Vergleich zu Peter Handkes Erzählung Wunschloses Unglück (1972) zieht, in der der Erzähler sieben Wochen nach dem Suizid seiner Mutter zu einer (literarischen) Reflexion ihres Lebens aufbricht, und zugleich darauf hinweist, dass La Place zunächst den Titel »Elemente einer Fami-

30 Eribon (Anm. 25), 20.

31 So auch der (ehemalige) Rapper McGarvey, der in seinem Buch Poverty Safari seinen Song Jump entsprechend erläutert: »Growing up, I never knew who to trust, looking at the world through the window of a school bus, gob-stopper in my mouth, I didn't mind school, it got me out the house. [...] Der Text ist autobiografisch und beschreibt meine Schulzeit sowie den plötzlichen Tod meiner Mutter.« (McGarvey [Anm. 19], 32f.).

32 Spoerhase (Anm. 21), 31. Zum Zusammenhang von Bildungsroman und Poetik sozialer Ungleichheit vgl. auch Steinmayr (Anm. 5).

33 Saša Stanišić, Herkunft, München 2019, 63. (Im Folgenden unter Angabe der Sigle »H« zitiert.).

In Herkunft ist es die Mutter, die die Rolle der Bildungsaufsteigerin innehat (»Sie hat, als Frau und aus einem nicht-akademischen Umfeld stammend, als einzige der drei Kinder studiert.«, H, 117), der jedoch, als der Vielvölkerstaat Jugoslawien auseinanderbricht, ein Klassenwechsel verwehrt bleibt: »Die ethnische Herkunft allerdings hing ihr wegen ihres arabischen Namens an wie ein hartnäckiges Gerücht. Sie war ein Makel in den Augen der neuen Bestimmer, ein Makel, der sich weder mit Ehrgeiz noch mit Bildung oder Geschick korrigieren ließ. [...] Herkunft ist das Zusammenzucken, wenn jemand in ihrer Geburtsstadt ihren Namen ruft.« $(\mathrm{H}, 117)$.

34 La Place ist auf Deutsch erstmalig unter dem Titel Das bessere Leben (1986; übersetzt von Barbara Scriba-Sethe) publiziert worden. Seit 2019 liegt es als Der Platz in einer Neuübersetzung von Sonja Finck vor.

Une femme ist in der deutschsprachigen Übersetzung von Regina Maria Hartig 1993 zunächst unter dem Titel Das Leben einer Frau und dann 2007 als Gesichter einer Frau erschienen. Auch Une femme wurde von Sonja Finck für den Suhrkamp Verlag neuübersetzt und unter dem Titel Eine Frau 2019 veröffentlicht. 
liensoziologie « tragen sollte, ${ }^{35}$ so macht dies einen größeren historischen Horizont und in systematischer Hinsicht den engen Konnex zwischen Tod, Familiengeschichte(n) und Lebensbeschreibung sichtbar. Unterstrichen wird dadurch aber auch die Möglichkeit der Verallgemeinerung der dargestellten Erfahrung sowie des damit verbundenen analytischen Anspruchs. In ihrem späteren autobiografischen Roman Les années (2008) setzt Ernaux dann »Familienerzählung und gesellschaftliche Erzählung « dezidiert in »eins. $\ll^{36}$

Auch sind für Autosoziobiografien solche autopoetologischen Reflexionen charakteristisch, in denen Fragen nach der Gattungszugehörigkeit ebenso erörtert werden wie die ihrer soziologischen Reichweite. Dabei wird insbesondere das Verhältnis von Erinnerung, Dokumentation und Fiktion von, wie es Stanišić in seinem noch jungen Buch zum Ausdruck bringt, »Herkunft « und poetischer ${ }$ Hervorbringung $^{37}$ problematisiert. Dies lässt sich auch bereits anhand von Ernaux' Erinnerungstexten beobachten. So denkt die Erzählerin in Eine Frau darüber nach, welches Textformat der Darstellung ihrer Mutter angemessen ist:

»Was ich zu schreiben hoffe, um ihr [der Mutter] gerecht zu werden, liegt vermutlich an der Nahtstelle von Familie und Gesellschaft, Mythos und Geschichte. Mein Vorhaben ist literarischer Art, denn es geht darum, nach einer Wahrheit über meine Mutter zu suchen, die nur durch Worte gefunden werden kann. [...] Gleichzeitig will ich sozusagen unterhalb dessen bleiben, was gemeinhin als Literatur gilt.« $(\mathrm{F}, 19)^{38}$

Wenn Ernaux ihr Buch - vermeintlich unpersönlich - Une femme nennt und zugleich mit den Worten »Meine Mutter ist gestorben [...]« $(\mathrm{F}, 9)$ beginnen lässt, so verdeutlicht sich darin der Anspruch, das Leben ihrer Mutter in einen größeren, sozialgeschichtlich relevanten Kontext zu stellen: Die Mutter soll nicht nur hinsichtlich ihrer »individuelle[n] Charakterzüge « betrachtet werden, vielmehr sollen diese zu »ihrer Lebensgeschichte und ihrer gesellschaftlichen Stellung « (F, 44; Hervorhebung von mir, E.B.) in Bezug gesetzt werden.

»Diese Form des Schreibens, die mir in die Richtung der Wahrheit zu gehen scheint, hilft mir, aus der Einsamkeit und Dunkelheit der individuellen Erfahrung herauszutreten, indem ich nach einer allgemeineren Bedeutung suche.« $(\mathrm{F}, 44)$

Von zentraler Bedeutung ist für Ernaux also zweierlei: Zum einen geht es ihr um das Anliegen, die Erfahrung sozialer Isolation, die mit einem Klassenwechsel einhergeht, erzählerisch zu bearbeiten, zu erklären und auf diese Weise abzuschwächen;

\footnotetext{
35 Eribon (Anm. 25), 134.

Aus Handkes Wunschloses Unglück zitiert auch Édouard Louis in seinem 2018 erschienenen Text Qui a tué mon père (Wer hat meinen Vater umgebracht, Frankfurt a.M. 2019, 26 u. 61).

36 Annie Ernaux, Die Jahre, Berlin 2017, 27.

37 »Herkunft, Hervorbringung, keine Heldengeschichten.« (H, 229); »Jetzt habe ich das Gefühl, als schriebe ich über meine Mutter, um sie dadurch zur Welt zu bringen.« (Annie Ernaux, Eine Frau, Berlin 2019, 36; im Folgenden unter Angabe der Sigle »F« zitiert.).

$38 \mathrm{Vgl}$. auch: »Dies ist keine Biographie und natürlich auch kein Roman, eher etwas zwischen Literatur, Soziologie und Geschichtsschreibung.« (F, 88).
} 
zum anderen soll die individuelle Geschichte bzw. die einzelne Familiengeschichte als symptomatische Erfahrung von »allgemeiner[ ] Bedeutung « sichtbar werden. Auf diese Form einer gleichermaßen individuellen wie überindividuellen Wahrheitssuche durch eine Tochter, in der die Mutter zuweilen den »Klassenfeind $(F, 55)$ erkennt, und auf das Problem der Repräsentation wird noch zurückzukommen sein. ${ }^{39}$

\section{III.}

\section{FOTOGRAFIEN ALS FAMILIEN- UND SOZIALGESCHICHTLICHE ZEUGNISSE}

Dass Eribon seinen Vater aus einer Fotografie herausgeschnitten hat und diesen Tilgungsakt nachträglich nicht nur aufklärt, sondern ihn für eine Auseinandersetzung mit seiner »unterwerfende[n] Subjektwerdung « (assujettissement) ${ }^{40}$ nutzt, weist auf die besondere Bedeutung hin, die Fotografien bei der autosoziobiografischen Erinnerungsarbeit zukommt. Die Rückkehr betrifft ja nicht nur den Ort und das Milieu der Herkunft, sondern findet auch in der Zeit statt, es ist eine Rückkehr in die eigene Kindheit - und dieser Umstand erfordert es, Vergangenes in der Gegenwart sichtbar zu machen. ${ }^{41}$ Es überrascht nicht, dass sich das Medium der Fotografie dafür in besonderem Maß eignet, dem insofern auch eine besondere familien- und sozialgeschichtliche Relevanz beigemessen werden kann. ${ }^{42}$ Die Foto-Ekphrasis dient den autosoziobiografischen Darstellungen dabei zugleich als Ausweis des Authentischen, als eine Art Realitätsanker. ${ }^{43}$ Die folgenden drei Beispiele, die sich medial sowie hinsichtlich ihrer historischen und nationalen Entstehungskontexte wesentlich unterscheiden, zeigen in ihrer Zusammenschau, auf welche Art und Weise Fotografien in Herkunfts- und Klassenübergangserzählungen integriert sind und für die Verknüpfung von Gegenwart und Zukunft sowie von individueller und >allgemeiner< Geschichte genutzt werden.

\footnotetext{
39 Vgl. Abschnitt IV.

40 Eribon (Anm. 25), 38.

41 In Die Jahre (frz. Les années, 2008) hat Annie Ernaux das Erzählen (ausgehend) von Fotografien zum bestimmenden Darstellungsprinzip gemacht. (Vgl. dazu auch Hanna Engelmeier, »Ernaux spricht für sich«, Texte zur Kunst, Schwerpunkt: Literatur [04.09.2019], abzurufen unter: https://www.textezurkunst. de/articles/annie-ernaux-spricht-fur-sich/ [18.02.2020]; sowie grundlegend zu den Foto-Texten Ernaux': Katharina Sykora, »Das Leben schreiben. Annie Ernaux’ Fotosoziobiografie«, Camera Austria International 146/2019, 9-20.).

42 »Die Rückbesinnung auf eine Familien- und Sozialgeschichte muss sich geradezu auf das Betrachten alter Fotografien stützen.« (Eribon [Anm. 25], 17.).

43 Auch Verlage nutzen - wie bereits aus Eribons Reflexion zum Foto auf der Titelseite der deutschen Ausgabe von Rückkehr nach Reims ersichtlich wird - dieses Prinzip: Fotografien der Autorin finden sich etwa auf allen Covern der deutschen (Neu-)Übersetzungen der Bücher Annie Ernaux', die im Suhrkamp Verlag erschienen sind. Gleiches gilt für Daniela Dröschers Zeige Deine Klasse. Die Geschichte meiner sozialen Herkunft (2018) sowie Christian Barons Ein Mann seiner Klasse (2020). Entsprechendes lässt sich für die Presse(arbeit) feststellen, wie die Illustration von Zeitungsartikeln zu den infrage stehenden Texten mit Fotografien der Autor*innen (als Kinder/Jugendliche) sowie oftmals auch von deren Eltern (oder Großeltern) zeigt (vgl. exemplarisch die Bebilderung zu Christian Baron, »Ein Mann seiner Klasse«, Freitag 10/2019; abrufbar unter https://digital.freitag.de/1019/ein-mann-seiner-klasse/ [16.02.2020]).
} 
Ray \& Liz

Richard Billinghams preisgekrönter Film Ray \& Liz kam im Frühjahr 2019 in die Kinos. Billingham, Jahrgang 1970, aufgewachsen in einem durch Armut geprägten Vorort Birminghams, war vor seinem Film vor allem für sein dokumentarisches Fotobuch Ray's a laugh (1996) bekannt. Darin porträtiert er seinen Vater und seine Mutter in Bildern aus den späten 1980er und frühen 90er Jahren. Zu sehen sind der alkoholkranke Ray und die adipöse, über und über tätowierte Liz, die Interieurs ihrer Wohnungen, ihre Beschäftigungen, ihre Haustiere, ihre Bekannten. Diesen dokumentarischen Blick auf seine Eltern erweiterte Billingham zunächst in Kurzfilmprojekten und dann mit Ray \& Liz um eine filmästhetische Ausdrucksform. Mit Ray \& Liz verfolge er dabei, so Billingham, das Ziel, »authentisch zu sein und das Leben zu zeigen, wie es ist, indem ich an die Orte zurückkehre, an denen all das geschehen ist. $\ll^{44}$

In struktureller Hinsicht nutzt der Kinofilm das Mittel der Verschränkung zweier Zeitebenen: Er beginnt mit dem älteren Ray, der, von einem Freund regelmäßig mit selbst hergestelltem Alkohol versorgt, in einem winzigen Zimmer seine Zeit verschläft, wenn er nicht gerade Radio hört oder aus dem Fenster schaut. Manchmal kommt Liz zu Besuch, die schon längst von ihm getrennt lebt, um ihn zu beschimpfen und um sein weniges Geld zu erleichtern. Diese Erzählebene stellt am Anfang, in der Mitte und am Ende des Films eine Gegenwart dar, die vor allem durch ihre völlige Handlungsarmut gekennzeichnet ist. In diese Rahmenhandlung sind zwei zeitlich deutlich früher angesiedelte Episoden eingefügt, die das frühere Leben von Ray und Liz als Ehepaar und Eltern von Richard und seinem jüngeren Bruder Jason zum Thema haben. Der soziale Abstieg der Familie wird unter anderem dadurch sichtbar, dass Lebensstandard und häusliche Umgebung immer schlechter werden: Zunächst bewohnt die Familie noch ein Reihenhaus, in der zweiten Episode sind sie in eine verwahrloste Hochhaussiedlung umgezogen. Das Ensemble der Kernfamilie wird in diesen Episoden durch einen behinderten Onkel ergänzt, der von einem Untermieter der Familie grausam gequält wird.

Eine besondere Bedeutung kommt in Ray \& Liz nun einer Fotografie zu, die zwar nur kurz, aber dafür gleich zweimal zu sehen ist: Am Ende der ersten Sequenz, in der der gealterte Ray in seiner Sozialwohnung trinkend und rauchend zu sehen ist, richtet sich die Kamera auf eine etwas verblichene Schwarz-Weiß-Fotografie vor einer weißen Bretterwand. Diese zeigt ein jüngeres Paar, Mann und Frau. Die Fotografie legt Zeugnis von der Vergangenheit von Ray und Liz ab und markiert zugleich den Beginn der narrativen Rückblende auf die Zeit, in der sie Ehepaar und Eltern waren. Jedoch zeigt die Fotografie gerade nicht die Schauspieler Justin

\footnotetext{
44 Elizabeth Fullerton, »Richard Billingham«, im Booklet zur DVD Ray \& Liz, o.S. Billingham kehrte für seinen Film auch ganz konkret an den Ort seiner Kindheit zurück. So bezieht die düster-dräuende, stark hoffnungslose und zugleich nüchterne, unsentimentale Atmosphäre von Ray \& Liz nicht nur sehr viel von der detaillierten, quasi-dokumentarischen Rekonstruktion der Wohnungsinterieurs seiner Kindheit, die längste Filmsequenz wurde zudem nahezu exakt an dem Ort gedreht, von dem er erzählt, nämlich in einer Wohnung schräg gegenüber derjenigen, in der Billingham als Kind mit seinen Eltern gewohnt hat (vgl. Björn Schneider, Verwahrlosung und Elend, abzurufen unter: https://www.cineman.ch/movie/2018/ RAYandLIZ/review.html [18.02.2020]).
} 
Salinger und Ella Smith, welche Ray (in seinen jüngeren Jahren) und Liz darstellen, sondern ein anderes, diesen durchaus ähnlich sehendes Paar. Anzunehmen ist, dass es sich dabei um eine authentische Aufnahme der Eltern Billinghams handelt.

Das retrograde Erzählen des Films wird also durch ein Foto eingeleitet, dass nicht nur in die Vergangenheit von Ray führt, sondern zudem auf die reale Vergangenheit und Kindheit des Filmemachers und dessen Eltern verweist. In der letzten Einstellung von Ray \& Liz ist dieselbe Fotografie nochmals zu sehen: Die Kamera schwenkt durch das nächtliche, rosa ausgeleuchtete Zimmer des betrunkenen Mannes und erfasst wiederum das Schwarz-Weiß-Bild. Gegenwart und Vergangenheit von Ray und seiner Familie sowie fiktionale Darstellung und Biografie des Filmemachers werden auf diese Weise eng miteinander verknüpft.

Was in Ray \& Liz freilich fehlt - und den Film von den meisten hier diskutierten Textbeispielen unterscheidet -, ist ein erwachsener Ich-Erzähler, der selbst als eine Figur in Erscheinung tritt, die zu ihrem Herkunftsmilieu zurückkehrt: Richard (Billingham) taucht im Film lediglich als Kind und Jugendlicher auf. Eine explizite autobiografische Perspektivierung der eigenen Kindheit aus der sozialen Distanz fällt damit aus. Hier sagt niemand: »Ich erzähle Euch meine Geschichte.« Kennt man Billinghams Biografie nicht, ist der Film daher nicht unmittelbar als autobiografisch grundierte Auseinandersetzung mit dem familiären und sozialen Milieu seiner Kindheit und Jugend erkennbar. Äußerungen des Filmemachers in Interviews und das Begleitmaterial zum Film, wie etwa das Booklet zur DVD, machen indessen diesen Hintergrund ganz deutlich. Auch die Frage, ob und wie ein >Entkommen aus dem Milieu, in das der >Zufall der Geburt< einen Menschen hineinversetzt, überhaupt möglich ist, wird in Ray \& Liz eher implizit als explizit beantwortet. Der Film zeigt die Intervention des Jugendamts, das die Unterbringung Jasons in einer Pflegefamilie veranlasst. Richard als älterer Bruder bleibt alleine in der Familie zurück. Das weitere Schicksal der Brüder wird nicht mehr erzählt.

\section{Herkunft}

Anders als bei Billingham, bei dem das Foto der noch jungen Eltern nicht in die Erzählhandlung eingebunden ist, sondern strukturell als Mittel der Abgrenzung und Verbindung der verschiedenen Zeitebenen der filmischen Erzählung, aber auch von Realität und Fiktion genutzt wird, sind in den hier zu diskutierenden literarischen Beispielen Fotografien Teil der Diegese. Interessant ist dabei insbesondere die jeweilige Fundsituation der Fotografien. In Stanišićs Herkunft entdeckt der Erzähler ein Bild in der Wohnung der Großmutter

»[i]m Barschrank hinter der Cognacflasche [...]. So gelagert, dass man es finden soll: ein Foto von Großvater, er sitzt auf einem Holzzaun (drei Planken, Pfeiler, drei Planken, Pfeiler), eine Berg-Lehne im Rücken. Es sind die Wälder und Wiesen von Oskoruša, der Gipfel von Vijarac. [...]

>Du hast nicht gefragt, ob du das darfst. - Was willst du denn?< Sie [die Großmutter] kam näher.

>Ich weiß es nicht, ich weiß nicht, was ich suche -«« (H, 107f.) 
Dem Ich-Erzähler wird schließlich klar, dass das Foto vom Großvater ihm deshalb irritierend bekannt vorkommt, weil es eine Szene zeigt, die seine Großmutter neun Jahre zuvor, nämlich 2009, auf einer Reise in das Heimatdorf ihres verstorbenen Mannes genau am Ort der älteren Aufnahme nachstellen ließ - mit ihm, dem unwissenden Enkel, an Stelle des auf dem Bild noch jungen Großvaters: »Großvater sitzt exakt dort auf dem Zaun, wo ich damals saß, als Großmutter mich mitgenommen hatte nach Oskoruša.« $(\mathrm{H}, 108)^{45}$

$\mathrm{Zu}$ dieser ersten und letzten von Enkel und Großmutter gemeinsam unternommenen Reise nach Oskoruša kehrt Herkunft immer wieder zurück; berichtet wird zudem, dass der Erzähler ein weiteres Mal mit seinen Eltern nach Oskoruša fährt - im April 2018 (vgl. H, 259-276), einige wenige Monate bevor er im Sommer 2018 neben verschiedenen Zeugnissen aus dem Leben seines Großvaters auch den Fund der Fotografie, die diesen auf dem Zaun in Oskoruša zeigt, macht. Unterbrochen wird die leitmotivische Darstellung der Rückkehr in das Dorf des Großvaters von Episoden, die die Kindheit des Erzählers in Višegrad behandeln, von Besuchen bei der immer stärker von der Demenz betroffenen Großmutter erzählen sowie von der Flucht mit den Eltern nach Heidelberg, von der Ankunft in Deutschland, von Freundschaften, Erfahrungen in der Schule und dem Erlernen der deutschen Sprache handeln. Die Gegenwart der Erzählung ist in Hamburg angesiedelt, wo Stanišić heute lebt. Mit seinem Erzählprinzip bricht Herkunft Linearität auf. Heimat wird pluralisiert zu Heimaten. ${ }^{46}$

An späterer Stelle - es wird der kurze, zweite Aufenthalt in Oskoruša berichtet - heißt es: »Ich will mir Großvater vorstellen als jungen Mann auf diesen Wegen. Im Sakko. Auf dem Esel. Es gelingt mir nicht, da ist nichts, da bin nur ich, der Fotos verwertet.« $(\mathrm{H}, 265)$ Das Imaginationsvermögen des Erzählers ist begrenzt. Im Gegensatz dazu verknüpft die im Barschrank der Großmutter gefundene Fotografie Herkünfte und Generationen deshalb miteinander, weil das Bild zum Teil einer Inszenierung wurde, die den ahnungslosen Ich-Erzähler zum Darsteller seines eigenen Großvaters machte. In Form einer unbewussten, aber äußerst physischen Verkörperung des Körpers des Großvaters tritt die eigene Familiengenealogie quasi geisterhaft vor das Auge des Protagonisten, als er der Fotografie ansichtig wird, und damit auch in die Vorstellungskraft der Lesenden ein. Zugleich wird durch die (Re-)Inszenierung in Oskoruša Kristinas spätere durch die zunehmende Demenz be-

\footnotetext{
45 An früherer Stelle in Herkunft findet sich die Szene der Aufnahme der jüngeren Fotografie in Oskoruša detailliert, was den Vorgang der Inszenierung durch die Großmutter (vgl. H, 80f.), aber auch bezeichnend beiläufig, was das Ergebnis betrifft, dargestellt: »Das Foto ist nicht weiter auffällig. Ein junger Mann im Hügelland. Das Haar verschwitzt, klebt an der Stirn. Ein Schaf liegt da. Der Berg ragt über seiner Schulter auf. Große Vögel kreisen um den Gipfel. Der Himmel ist rigoros blau.« $(\mathrm{H}, 81)$.

46 Dieses Bestreben steht im Kontext eines spezifischen Vorbehalts gegenüber der Rückkehr zu einer wie auch immer definierten sozio-kulturellen Herkunft: Das »Widerstreben« des Erzählers von Herkunft richtet sich nämlich gerade »gegen die Fetischisierung von Herkunft und gegen das Phantasma nationaler Identität. Ich war für das Dazugehören.« $(\mathrm{H}, 216) \mathrm{Vgl}$. dazu auch: »Ich begann mich mit meiner Herkunft zu beschäftigen, gab es aber lange nicht zu. Es erschien mir rückständig, geradezu destruktiv, über meine oder unsere Herkunft zu sprechen in einer Zeit, in der Abstammung und Geburtsort wieder als Unterscheidungsmerkmale dienten, Grenzen neu befestigt wurden und sogenannte nationale Interessen auftauchten aus dem trockengelegten Sumpf der Kleinstaaterei. In einer Zeit, als Ausgrenzung programmatisch und wieder wählbar wurde.« $(\mathrm{H}, 62)$.
} 
dingte Verwechslung ihrer Angehörigen auf gewisse Art vorweggenommen. Denn in beiden Fällen findet eine Überblendung der Toten und der Lebenden statt und damit ein Ineinanderschieben der Generationen.

\section{La Place}

Den beiden bereits diskutierten Beispielen Ray \& Liz und Herkunft aus dem Jahr 2019 lässt sich mit Ernaux’ Vaterbuch aus der ersten Hälfte der 1980er Jahre eine weitere, zeitlich frühere literarische Foto-Ekphrasis hinzufügen. In Der Platz spielt eine Fotografie gleich zu Beginn eine signifikante Rolle. Ähnlich wie in Herkunft wird diese nicht nur zum Vehikel einer Reise in die Vergangenheit der eigenen Familienbiografie, sondern dient zugleich dazu, die Generationen als gleichermaßen voneinander entfernt als auch miteinander verbunden in Erscheinung treten zu lassen.

Der Platz setzt mit den Erledigungen ein, die durch den Tod und die anstehende Beerdigung des Vaters bedingt sind:

»Wir suchten die Kleider meines Vaters zusammen, um sie an Leute weiterzugeben, die sie brauchen konnten. In seiner Alltagsjacke, die in der Kammer hing, fand ich seine Brieftasche. Darin ein wenig Geld, seinen Führerschein und in dem aufklappbaren Teil ein altes Foto in einem zusammengefalteten Zeitungsartikel. Das Foto mit gezackten Rändern zeigte eine Gruppe von Arbeitern verteilt auf drei Reihen, alle blickten in die Kamera, alle hatten eine Mütze auf. Ein typisches Foto aus dem Geschichtsbuch, zur Illustration eines Streiks oder der Volksfront. Ich erkannte meinen Vater in der hintersten Reihe, mit ernster, fast sorgenvoller Miene. Viele lachen. Der Zeitungsartikel listete die Ergebnisse der Aufnahmeprüfung an der Fachhochschule für Grundschullehrerinnen auf, nach Noten sortiert. Mein Name stand an zweitoberster Stelle. «77

Hier wird die Bedeutung von Fotografien als Wegmarken auf der Reise zur sozialen Herkunft ganz deutlich. Dass die Lebenswege von Vater und Tochter miteinander verschränkt und aufeinander bezogen sind, kommt durch den Umstand zum Ausdruck, dass das Foto des Vaters als Angehöriger der Arbeiterklasse in einen Zeitungsartikel, der den Bildungsaufstieg der Erzählerin belegt, hineingelegt wurde.

Bezeichnenderweise wird durch diesen doppelten Fund eine Reflexion über die Frage nach der >richtigen< Repräsentation des Vaters und seines Lebens durch die Tochter angeregt. Es schließt sich folgende Textpassage an, die hier trotz ihrer Länge ausführlich zitiert wird, weil sie in wenigen Absätzen Bemerkenswertes leistet:

»Auf der Zugfahrt am Sonntag versuchte ich, meinen Sohn zu beschäftigen, damit er ruhig blieb, die Reisenden der ersten Klasse mögen keinen Lärm und keine herumlaufenden Kinder. Mit einem Mal, fassungslos >jetzt gehöre ich wirklich zum Bürgertum< und >es ist zu spät<.

Später, im Laufe des Sommers, während ich auf meine erste Stelle wartete, >ich sollte das alles erklären $<$ Ich wollte alles sagen, über meinen Vater schreiben, über sein Leben und über die Distanz, die in meiner Jugend zwischen ihm und

\footnotetext{
47 Annie Ernaux, Der Platz, Berlin 2019, 18. (Im Folgenden unter Angabe der Sigle »P« zitiert.).
} 
mir entstanden ist. Eine Klassendistanz, die zugleich aber auch sehr persönlich ist, die keinen Namen hat. Eine Art distanzierte Liebe.

Daraufhin begann ich einen Roman zu schreiben, mit ihm als Hauptfigur. Mittendrin ein Gefühl des Ekels.

Seit Kurzem weiß ich, dass der Roman unmöglich ist. Um ein Leben wiederzugeben, das der Notwendigkeit unterworfen war, darf ich nicht zu den Mitteln der Kunst greifen, darf ich nicht >spannend< oder >berührend< schreiben wollen. Ich werde die Worte, Gesten, Vorlieben meines Vaters zusammentragen, das, was sein Leben geprägt hat, die objektiven Beweise seiner Existenz, von der ich ein Teil gewesen bin.

Keine Erinnerungspoesie, kein spöttisches Auftrumpfen. Der sachliche Ton fällt mir leicht, es ist derselbe Ton, in dem ich früher meinen Eltern schrieb, um ihnen von wichtigen Neuigkeiten zu berichten.«(P, 19f.)

Mit dem Sohn wird eine weitere Generation in die Darstellung einbezogen. Die »Klassendistanz« zum Vater spiegelt sich in der Reise in der ersten Zugklasse: Für den Sohn womöglich schon eine Selbstverständlichkeit, führt sie die Mutter zu der erschreckenden Erkenntnis, dass sie ihre Klassenzugehörigkeit unumkehrbar gewechselt hat. Die Rückkehr zur Herkunft kann sich nur noch in der Erzählung vollziehen, für die sich nun das Problem der adäquaten Form stellt. Der favorisierte Ton ist mit demjenigen der brieflichen Kommunikation mit den Eltern identisch: »sachlich «, vielleicht aber auch eher $»$ simpel ${ }^{48}{ }^{48}$ Die erworbene soziale und sprachliche Distanz wird dadurch stilistisch ein Stück weit eingeebnet, ein Erheben, ein »spöttisches Auftrumpfen« des Bürgertums gegenüber der Arbeiterklasse gilt es zu vermeiden. Der Klassenabstand, der zwischen Vater und Tochter, Erzählerin und Erzählobjekt herrscht, darf sich nicht im Ausdruck, nicht im discours niederschlagen, obwohl zugleich genau dieser Gegenstand der Erzählung ist.

Doch an wen richtet sich La Place eigentlich? Wem möchte die Erzählerin etwas »erklären«? Dem (verstorbenen) Vater die »distanzierte Liebe« oder einem bürgerlichen Lesepublikum die Erfahrung der Klassendistanz aus der Perspektive der Bildungsaufsteigerin?

\section{IV.}

\section{DAS PROBLEM DER REPRÄSENTATION UND DIE FRAGE DER ADRESSIERUNG}

Das Narrativ von der Rückkehr zur sozialen und familiären Herkunft ist mit einem weiteren Problem konfrontiert: der Gefahr einer - mit einem Wort Billinghams - »Ausbeutung (Exploitation) « ${ }^{49}$ der eigenen Eltern und Großeltern. Billingham

\footnotetext{
48 In der älteren deutschen Übersetzung von La Place wird der von Ernaux verwendete Ausdruck »[1]'écriture plate« (Annie Ernaux, La Place, Paris 2010, 18) mit »simple Schreibweise « übersetzt (Annie Ernaux, Das bessere Leben. Erzählung, Frankfurt a.M. 1988, 17).

49 Richard Billingham, » I just hated growing up in that tower block $<$. Interview mit Tim Adams «, in: Guardian (13.03.2016); abrufbar unter: https://www.theguardian.com/artanddesign/2016/mar/13/richardbillingham-tower-block-white-dee-rays-a-laugh-liz (08.02.2020).
} 
spricht von der Notwendigkeit einer warmen Empathie, Ernaux von Liebe, die die >Ausbeutung< auszugleichen hätte. Die Frage nach der richtigen Haltung gegenüber dem Erzählten, nach einem angemessenen Ton der Darstellung wird auch von anderen Autosoziobiografen thematisiert. So schreibt Édouard Louis in Wer hat meinen Vater umgebracht:

»Auch das habe ich bereits erzählt - aber ich muss mich doch wiederholen, wenn ich von deinem Leben erzähle, denn von einem solchen Leben will niemand hören! Man muss sich doch wiederholen, bis sie uns zuhören! Um sie zum Zuhören zu zwingen! Wir müssen doch eigentlich schreien! [...] Ich scheue mich nicht, mich zu wiederholen, denn was ich schreibe, was ich erzähle, folgt nicht den Erfordernissen der Literatur, sondern denen der Notwendigkeit, der Dringlichkeit, denen des Feuers. $\ll^{50}$

Louis wählt das narrative Mittel der Wiederholung und das Pathos der Anklage, um das Leiden und Leben seines Vaters einem imaginären Publikum, das davon nichts hören will, nahezubringen. Die letzten Worte seines Textes legt er seinem Vater in den Mund: »Recht so, ich glaube, was es bräuchte, das ist eine ordentliche Revolution. $\ll^{51}$ Ähnlich verfährt auch Eribon in Rückkehr nach Reims, wenn er in einer performativen Geste ein affiziertes Sprechen für seine Form der Darstellung wählt. ${ }^{52}$ Auf diese Weise kommt es zu einer »Wiederaneignung eines Merkmals plebejischen Sprechens [...], der unsublimierten Aggressivität «. ${ }^{53}$

Diese Form der Darstellung und ihr Erfolg können provozieren, wie etwa einer Einschätzung der Literaturkritikerin Iris Radisch zu entnehmen ist, die Louis und Eribon »Vulgärsoziologie« vorwirft:
»Den Hochmut, mit dem die beiden Soziologen (Louis studierte bei Eribon an der École normale supérieure) ihre hilf- und sprachlosen Eltern am Nasenring der internationalen Literaturöffentlichkeit vorführten, sah man ihnen nach, zu erfreut war man über die Nachrichten von einem fremden Stern und seinen kulturlosen Eingeborenen, die es so selten auf die Hauptbühne der Literatur schaffen. $\ll^{54}$

Eher im Undeutlichen bleibt hier, inwiefern sich der Hochmut und die Überheblichkeit, von der die Darstellung der Eltern bei Eribon und Louis gekennzeichnet sei, anhand von deren Texten festmachen lässt. Die Kritik umfasst dabei auch die Reaktionen des Lesepublikums, das offenbar als eines vorgestellt wird, das die Arbeiterklasse aus eigener biografischer Erfahrung nicht kennt. Es kann hier nicht darum gehen, die genannten Autoren, ihre Texte und deren Leser*innen gegenüber solchen Einschätzungen in Schutz zu nehmen. Allerdings ist diese Form der Kritik

\footnotetext{
50 Louis (Anm. 35), 20.

51 Louis (Anm. 35), 77.

52 Vgl. dazu Blome (Anm. 27), 57.

53 Dirck Linck, »Die Politisierung der Scham. Didier Eribons Rückkehr nach Reims«, Merkur 808 (Sept. 2016), 34-47, hier: 42.

54 Iris Radisch, »Macron beklaut meinen Vater«, DIE ZEIT 5/2019 (24.01.2019), abrufbar unter https:// www.zeit.de/2019/05/vater-umgebracht-edouard-louis-buch-frankreich-gelbwesten (09.02.2020).
} 
eventuell in symptomatischer Hinsicht von Interesse. Denn vielleicht verkehrt sich in ihr gerade die Problematik der erworbenen Klassendistanz und die Verschränkung von persönlicher Erzählung und Gesellschaftsanalyse, die in den Texten der Klassenübergänger ihren Ausdruck findet, zum Vorwurf, wenn die Schreibweisen von Eribon und Louis mit Konzepten wie dem des »Pamphlet[s] « und der »Drastik «55 in Verbindung gebracht werden sowie deren Indienstnahme für politische Anliegen (insbesondere der der so genannten »Gelbwesten«) beklagt wird. Auf diese Weise verschränken sich ethische, politische und ästhetische Beurteilungsaspekte solcherart miteinander, dass es schwer wird, deren implizit bleibenden Zusammenhang klar zu erfassen. ${ }^{56}$ Festgestellt werden kann jedoch an den infrage stehenden Texten, dass die Erzähler es sich mit der Darstellung der Eltern nicht allzu leicht machen; das Ringen um die richtige Selbst- und Fremdrepräsentationsweise ist ihnen in unterschiedlicher Form eingeschrieben: Louis kennzeichnet den monologischen Dialog mit seinem Vater, in dem nur er als Sohn spricht, gleich zu Beginn von Wer hat meinen Vater umgebracht als »für beide brutal: Dem Vater bleibt verwehrt, seine eigene Lebensgeschichte zu erzählen, und der Sohn ersehnt sich eine Antwort, die er niemals erhalten wird. ${ }^{57}$ Und auch Eribon schwankt zwischen dem von ihm behaupteten Privileg des Wissens des Bildungsaufsteigers und Soziologen, der die Sprachund Reflexionslosigkeit seiner Herkunftsklasse hinter sich gelassen hat und nun eben diese analysiert und darstellt, und der Einsicht in genau diese Mechanismen und der mit ihnen verbundenen Hybris. ${ }^{58}$ Pointiert findet sich diese Problematik bereits lange vor den Auseinandersetzungen von Eribon und Louis bei Ernaux formuliert:

»Beim Schreiben ein schmaler Grad zwischen der Rehabilitierung einer als unterlegen geltenden Lebensweise und dem Anprangern der Fremdbestimmung, die mit ihr einhergeht. Weil diese Art zu leben unsere war, sogar unser Glück, sie aber auch die demütigenden Grenzen unseres Daseins bestimmte (das Bewusstsein, dass es >bei uns zu Hause nicht gut genug war<), will ich gleichzeitig über das Glück und die Fremdbestimmung schreiben. Allerdings habe ich eher

\footnotetext{
55 Radisch (Anm. 54).

56 Interessanter Weise nimmt Radisch an anderer Stelle Annie Ernaux von der hier zitierten Kritik folgendermaßen aus: »[...] anders als Eribon, der in seinen Büchern überheblich mit seinen Proletariereltern abrechnet, liebt Annie Ernaux ihre Eltern und hat die Flucht aus der sozialen Klasse nie überwunden. Noch mit 78 Jahren fühlt sie sich weder in ihrem Herkunftsmilieu noch in der französischen Kulturelite, die ihre Bücher verlegt und liest, zu Hause.« (Iris Radisch, »Ich fühle mich schuldig «, DIE ZEIT 42/2018 [11.10.2018]).

57 Louis (Anm. 35), 7 f. Dass der Erzähler keine Antwort von seinem Vater erhält oder auch nur erwartet, liegt hier nicht etwa daran, dass dieser schon verstorben wäre, auch wenn er, »gerade mal über fünfzig «, zu denjenigen Menschen gehört, »für die die Politik einen verfrühten Tod vorgesehen hat« (13). Vielmehr wird die Entfremdung des Sohnes vom Vater ursächlich für die Kommunikationsunmöglichkeit und die damit zusammenhängende Darstellung im Präteritum angeführt - bezeichnenderweise wird diese Auskunft, die den eigenen Redeschwall kommentiert und analysiert, in Klammern gesetzt: »(Ich rede in der Vergangenheitsform von dir, weil ich dich nicht mehr kenne. Das Präsens wäre verlogen.)« (40).

58 Vgl. z. B.: »Von Arbeitermilieus wird nicht oft gesprochen. Und wenn, dann meistens unter der Maßgabe, dass derjenige, der spricht, sie verlassen hat und dass er von seinem >Aufstieg<, über den er froh ist, berichten will. Die soziale Illegitimität der Beschriebenen wird genau in dem Moment bestätigt, wo jemand, der die notwendige kritische Distanz (und damit eine wertende, urteilende Perspektive) erreicht hat, sie beschreibt und anklagt.« (Eribon [Anm. 19], 90.).
} 
das Gefühl, zwischen den beiden Seiten dieses Widerspruchs hin- und herzuschwanken.« $(\mathrm{P}, 45)$

Die Rehabilitation, von der hier die Rede ist, lässt sich im Sinne der ursprünglichen lateinischen Bedeutung von rehabilitatio als eine Art literarische >Wiederherstellung< oder >Hervorbringung ${ }^{59}$ eines $>$ gemeinsamen $<$ Daseins verstehen. Autosoziobiografien versuchen paradoxerweise also nicht zuletzt, in der Darstellung von Klassendistanz Klassengegensätze erzählerisch zu überwinden. Natürlich darf - nicht zuletzt auch mit Blick auf diejenigen autosoziobiografischen Texte, die im Gefolge von Ernaux, Eribon und Louis in den letzten Jahren in Deutschland oder anderen Ländern erschienen sind, - die Frage gestellt werden, ob dies den einzelnen Autosoziobiografien tatsächlich gelingt. Anerkannt werden muss dabei jedoch, dass die Antwort womöglich auch von den individuellen Lebens- und Klassenerfahrungen derjenigen abhängt, die sich um eine politische, literaturkritische oder literaturwissenschaftliche Einschätzung autosoziobiografischer Darstellungsweisen bemühen.

\section{V.}

\section{AUF DEM WEG ZU EINER >TRANSCLASSE<-COMMUNIO?}

Die analytisch-narrative Auseinandersetzung mit der eigenen Herkunft vor dem Hintergrund einer Entfremdungserfahrung geht mit einer spezifischen Sehnsucht nach milieuübergreifenden Orten und Formen der Vergesellschaftung einher. In Eribons Rückkehr nach Reims erfüllen beispielsweise die cruising areas der schwulen Community von Paris diese Funktion. Aber auch dem Kirchenchor kann eine solche Funktion zukommen ${ }^{60}$ oder - wie zu Beginn von Ladj Lys Film Les Misérables der klassennivellierenden Fußballbegeisterung.

Die Suche nach solchen Orten und Gemeinschaften wird durch die Erfahrung einer sozialen Isolation hervorgerufen, die mit dem Klassenwechsel einhergeht. So schreibt Ernaux in Eine Frau:

»Meine Mutter, die in ein beherrschtes Milieu hineingeboren worden war, das sie hinter sich lassen wollte, musste erst Geschichte werden, damit ich mich in der beherrschenden Welt der Wörter und Ideen, in die ich auf ihren Wunsch hin gewechselt bin, weniger allein und falsch fühle.« (F, 88f.)

\footnotetext{
59 Vgl. Anm. 37.

60 »Im gemischten Kirchenchor sang der Arzt neben dem Bauern, neben dem Arbeitslosen, neben dem rumänischen Vorarbeiter, neben der Sozialarbeiterin. In temporären Gemeinschaften wie diesen lockert sich das Korsett des Ökonomischen. Sie wecken die Sehnsucht nach communio, die ein jeder in sich hat ein kommunistisches Begehren (Bini Adamczak). (Daniela Dröscher, Zeige deine Klasse. Die Geschichte meiner Herkunft, Hamburg 2018, 71.).
} 
Die Filme und Texte, die hier zur Diskussion stehen, können also schließlich auch als Versuche gewertet werden, durch das Erzählen Einsamkeit und Entfremdung zu überwinden. ${ }^{61}$

Da derlei Versuche aber inzwischen so zahlreich sind, entsteht zugleich noch eine andere, neue, nämlich durch gegenseitige Lektüren und Intertextualität gestiftete Form der Vergemeinschaftung. Die autosoziobiografische Darstellung bietet offenbar ein Identifikationspotenzial, das Erzählungen von weiteren Lebens- und Bildungsgeschichten als Analyse von Sozial- und Generationenverhältnissen befördert.

So lassen sich, wie bereits gezeigt, Rückkehr nach Reims eine Vielzahl an Texten und Filmen zur Seite stellen, die ebenfalls aus der Perspektive des individuellen sozialen Aufstiegs aktuelle gesellschaftliche Verhältnisse verhandeln. Einige dieser Autosoziobiografien weisen sich dabei als explizit von Eribon inspiriert aus. Dies gilt beispielsweise für Édouard Louis' Texte, aber auch für Daniela Dröschers Zeige deine Klasse (2018). ${ }^{62}$

Darüber hinaus ist der von Eribon geschilderte Klassenwechsel inzwischen selbst schon wieder Gegenstand von Analysen der Prozesse und Narrationen des sozialen Aufstiegs geworden. Anzuführen ist hier insbesondere Chantal Jaquets hinsichtlich der Figur des Klassenübergängers überaus einschlägiger Essay Les transclasses, ou la non-reproduction (2014). Jaquet stellt Rückkehr nach Reims in den Zusammenhang mit einer ganzen Reihe weiterer autosoziobiografischer Texte (u.a. von Annie

61 Zum Zusammenhang von Bildungs(erfolg), Entfremdungserfahrung und sozialer Exklusion vgl. Steinmayr (Anm. 5), 104-106.

$62 »$ Erst [...] als ich Eribon las, verstand ich, dass ich in Hinblick auf mein Herkunftsmilieu eine Scham zweiter Ordnung gelebt hatte. Sie stammte nicht ursächlich aus mir, sie war mir von meinem Vater, dem emporgekommenen Bauernkind, und seiner Mutter, meiner Großmutter, vererbt worden. « (Dröscher [Anm. 60], 23.).

Außerdem wird Rückkehr nach Reims bereits als eine Art divers perspektivierte mise en abyme in literarischen Erzählungen von der Rückkehr zur sozialen Herkunft involviert, wobei zudem die Frage der Adressierung autosoziobiografischer Texte beiläufig beantwortet wird. Vgl. dazu etwa folgende Textstelle aus Bjergs Serpentinen, in der von einer (nicht-)geteilten Leseerfahrung des Ich-Erzählers, einem zum Soziologieprofessor (!) aufgestiegenen Arbeiterkind, und seinem schwulen Jugendfreund Micha, einem Bauern, erzählt wird:.

»Er [Micha] ging zum Klavier, griff ein Buch und wedelte damit. Ich kannte das Buch. [...] Ich sagte: >Arbeiterjunge schafft es auf die Uni. Gehört nicht mehr dahin, wo er herkommt. Gehört nicht dahin, wo er jetzt ist.<.

Micha sagte: >Junger Schwuler zieht in die Großstadt. Gehört nicht mehr dahin, wo er herkommt. Gehört nicht dahin, wo er jetzt ist.<.

Ich sagte: >Da haben wir zwei verschiedene Bücher gelesen.< [...].

Micha sagte: >Macht ja nichts.<.

Ich sagte: >Nein, warum sollte das was machen.<.

Micha sagte: >Ich hab mich nicht getraut, im Laden nach dem Buch zu fragen. Ich wusste nicht, wie man den Namen ausspricht. Oder den Titel. Ich hätte das Buch fast nicht gelesen, weil ich kein Französisch kann. Witzig, oder?<.

Ich: >Hättest du was verpasst?<.

Micha lachte: >Dass die Studierten so ein Getue veranstalten, wusste ich vorher. Und den Rest sowieso.<.

Ich sagte: >Wir sind nicht die Zielgruppe.« (Bov Bjerg, Serpentinen, Berlin 2020, 100; Hervorhebung von mir, E.B.).

Für unwahrscheinlich halte ich, dass diese Textstelle nicht auf Rückkehr nach Reims, sondern, wie Felix Stephan annimmt, auf Édouard Louis' Das Ende von Eddy anspielt (vgl. Felix Stephan, »Aufstiegsangst«, Süddeutsche Zeitung 1./2.02.2020, 18). 
Ernaux, Richard Hoggart, Jack London, Paul Nizan, Stendhal, Richard Wright und John Eggar Wideman). Anhand dieser vollzieht Jaquet die »Komplexion« sozialer Transgressionsphänomene nach. ${ }^{63}$ Aus dem Blickwinkel des Individuums, dem trotz einer ungünstigen sozialen Ausgangssituation ein gesellschaftlicher Aufstieg gelingt, wird laut Jaquet dabei die Regel von der Reproduktion von Sozialverhältnissen (u.a. durch Bildung) gerade nicht widerlegt, sondern vielmehr bestätigt: Denn Klassenstrukturen machen sich immer dann besonders deutlich bemerkbar, wenn es zur Überschreitung von Klassengrenzen kommt. Jaquets Analyse fehlt dabei nicht der autobiografische Hinweis auf die eigene Herkunft aus armen und provinziellen Verhältnissen, wodurch auch der Lebensweg der Autorin, heute Philosophieprofessorin an der Sorbonne, als weitere >Ausnahmegeschichte< einer Bildungsaufsteigerin und Klassenübergängerin lesbar wird. ${ }^{64}$

Wie an Jaquets umfassender Darstellung offensichtlich wird, ist Eribons Rückkehr nach Reims sicher nicht als maßgeblicher oder gar alleiniger Kristallisationspunkt anzusehen, von dem aus das Erzählen der eigenen Herkunftsgeschichte aus der Perspektive einer erworbenen sozialen Distanz und die Schaffung eines transclasseGenres seinen Ausgang nimmt. Vielmehr muss in dieser Hinsicht zweierlei ins Auge gefasst werden: Erstens ist festzustellen, dass es ein anderes maßgebliches Zentrum einer gleichermaßen theoretischen wie lesebiografischen Verankerung gibt, mit dem der eigene Lebensweg in einer Vielzahl der hier zur Diskussion stehenden Texte in gesellschaftsanalytischer Perspektive ausgeleuchtet wird: Pierre Bourdieu, in dessen Person sich die biografische Erfahrung des Klassenwechsels mit soziologischer Expertise verbindet. Dies stellt Eribon in aller Ausführlichkeit und Deutlichkeit heraus. Es lässt sich bei Louis beobachten und schreibt sich - mit Rekurs auf Eribon - im Text Dröschers fort. ${ }^{65}$ Und nicht zuletzt nimmt der Essay Jaquets seinen Ausgang von (einer kritischen Auseinandersetzung mit) Bourdieu. ${ }^{66}$ Aber auch bei Autor*innen, die ihre autosoziobiografischen Texte bereits vor Eribons Rückkehr nach Reims verfasst haben, finden sich entsprechende Verweise. Dies gilt natürlich in besonderem

\footnotetext{
63 Jaquet fasst »Komplexion« als »die Kette der Bestimmungen, die sich zur Textur eines singulären Lebens verknüpfen«, auf (vgl. Chantal Jaquet, Zwischen den Klassen. Über die Nicht-Reproduktion sozialer Macht. Mit einem Nachwort von Carlos Spoerhase, Konstanz 2018, 101). Komplexion sei »weniger der Ausdruck einer Form als einer Formation. [...] Doch wenn sich die Komplexion des Klassenübergängers als Einheit der körperlichen und geistigen Charakteristika definieren läßt, die unter Einwirkung einer vielfältigen Kausalität miteinander verschlungen sind, bleibt die Natur dieses Flechtwerks von Gegebenheiten noch zu klären.« (103).

Diese Bestimmung Jaquets von Komplexion weist eine gewisse Nähe zu dem aus den Gender Studies stammenden Forschungsparadigma der Intersektionalität auf, auch wenn Jaquet weniger stark die Interdependenz verschiedener sozialer Differenzkategorien bei der Konstitution eines inter- und intrasektional bestimmten Subjekts betont. (Vgl. dazu Eva Blome, »Erzählte Interdependenzen. Überlegungen zu einer kulturwissenschaftlichen Intersektionalitätsforschung«, in: Peter C. Pohl, Hania Siebenpfeiffer [Hrsg.], Diversity Trouble. Vielfalt - Gender - Gegenwartskultur, Berlin 2016, 45-67 sowie in Anwendung auf Rückkehr nach Reims Blome [Anm. 27], 51-60.).

64 Vgl. Jaquet (Anm. 63) 61 u. 87f. (sowie den Hinweis zur Biografie der Autorin im Klappentext der deutschsprachigen Ausgabe, o.S.).

65 Vgl. Édouard Louis, »Wer sie beleidigt, beleidigt meinen Vater«, ZEIT online, 05.12.2018, abrufbar unter https://www.zeit.de/kultur/2018-12/gelbwesten-frankreich-gesellschaft-sozialitaet-klassen-gewaltedouard-louis (08.02.2020) sowie Dröscher (Anm. 60), 24 u. 30.
}

66 Vgl. dazu auch Spoerhase (Anm. 21), 27 f. u. 30 sowie ders. (Anm. 22), 237-241. 
Maße für die Texte von Annie Ernaux, die, wie bereits offensichtlich geworden sein dürfte, zusammen mit Eribon und Louis eine Art französisches Dreigestirn autosoziobiografischen Schreibens bildet und in diesem, gleichermaßen durch ein Meisterinnen-Schüler-Verhältnis ${ }^{67}$ wie durch eine hochgradige Intertextualität getragenen, Verbund die älteste von drei Generationen autosoziobiografischen Schreibens repräsentiert, aber eben auch zugleich mit nur 10 Jahren Altersunterschied nahezu derselben Generation wie Pierre Bourdieu angehört. ${ }^{68}$ Insofern es hier um die Stiftung einer Wahlverwandtschaft geht, die auch in den deutschen Kosmos autosoziobiografischen Schreibens ausstrahlt, könnte man Bourdieu daher als soziologischen Patriarchen dieser Konstellation ansehen. Er ist dabei auch für die Aufklärung der individuellen Risiken und Gefahren, die sich mit einem Klassenwechsel verbinden, von Bedeutung.

Welche Relevanz Bourdieu für das Erzählen von der Herkunft aus prekären sozialen Verhältnissen zukommt, illustriert besonders anschaulich Thomas Melles Buch Die Welt im Rücken (2016), das sich autopoetologisch als »eine Art negative MiniKulturgeschichte « und als »Anti-Bildungsroman « ausweist. ${ }^{69}$ An dessen Ende deutet der Autor an, dass seine manisch-depressive Erkrankung, deren Schilderung den größten Teil seiner Darstellung einnimmt, womöglich auf seinen Bildungsaufstieg zurückzuführen ist. ${ }^{70}$ Zuvor heißt es über einen Besuch bei seiner Mutter und in seiner ehemaligen Schule: »Ich fahre nach Bonn. Ich hasse Bonn so sehr, und doch muss ich hin. Das Buch über meine Herkunft kann ich erst in Jahrzehnten schreiben, wenn überhaupt. « ${ }^{71}$ In Die Welt im Rücken ersetzt also die Reise an den Herkunftsort, die mit Erinnerungen an seine Kindheit und Schulzeit einhergeht, die umfassendere

67 Vgl. dazu »>Ich möchte, dass die Worte genauso hart sind wie das Leben $<$ Interview von Gabriella Herpell mit Annie Ernaux«, Süddeutsche Zeitung Magazin 43 (24.10.2019), 22-29, hier: 22.

68 Vgl. Annie Ernaux, »Bourdieu: le chagrin«, LE MONDE 05.02.2002; abzurufen unter: http://www. homme-moderne.org/societe/socio/bourdieu/mort/aernau.html (08.02.2020); als »un choc ontologique violent« kennzeichnet Ernaux hier die Lektüre der Bücher Les hérities: les étudiants et la culture und La Reproduction. Éléments piur une théorie du système d'enseignement, die Bourdieu zusammen mit JeanClaude Passeron verfasste und die 1964 und 1970 erschienen sind (dt. Die Erben. Studenten, Bildung und Kultur, 1970, und Grundlagen einer Theorie der symbolischen Gewalt. Kulturelle Reproduktion und soziale Reproduktion, 1973). Vgl. aber auch Ernaux’ Äußerung: »Ich schulde Bourdieus Soziologie alles Mögliche, aber ich arbeite nicht >nachく Bourdieu. « (Annie Ernaux, Le vrai lieu. Entretiens avec Michelle Porte, Paris 2014, 73; zit. nach der Übersetzung von Hanna Engelmeier, in: Dies. [Anm. 41]).

Besonders einflussreich und interessant im Kontext der im vorliegenden Aufsatz verhandelten (deutschsprachigen) autosoziobiografischen Darstellungen sind außerdem Bourdieus Hauptwerk Die feinen Unterschiede (La distinction. Critique sociale du jugement, 1979), insbesondere sein u. a. dort entwickeltes Habituskonzept, sowie Die biographische Illusion (L'illusion biographique, 1986) und Ein soziologischer Selbstversuch (Esquisse pour une auto-analyse, 2001).

69 Thomas Melle, Die Welt im Rücken, Berlin 2016, 339.

70 »Wie soll denn das auch klappen: Ein Arbeiterkind aus schwierigen Verhältnissen wird von den Jesuiten intellektuell aufgepimpt, von Nabokov ins Schöngeistige verschickt und vom Studium ins Nichtmehrvorhandensein theoretifiziert, und was soll das dann, die Genetik noch im Nacken, ein Dichter werden, oder wie, ein glücklicher Mensch? Hört mir auf.« (Melle [Anm. 69], 344); vgl. auch: »Und die Frage, was eigentlich die Gesellschaft zu meiner Erkrankung beigetragen hat, und das Wissen, diese Frage nur immer genauer stellen, nie beantworten zu können.« (Melle [Anm. 69], 333) sowie bereits an früherer Stelle im Text: »Die Herkunft und ihre Konsequenzen sind einfach nicht abzuschütteln.« (Melle [Anm. 69], 275).

71 Melle (Anm. 69), 183; Hervorhebung von mir, E.B. 
erzählende Auseinandersetzung mit der sozialen und familiären Herkunft. ${ }^{72}$ Der IchErzähler kann (noch) keine Autosoziobiografie schreiben. Warum aber nicht? Womöglich fehlt ihm, der bei seiner alleinerziehenden Mutter aufgewachsen ist, dazu schon in ganz grundlegender Weise der prototypische Ausgangs- und Reibungspunkt autobiografischen Erzählens (von Männern): nämlich der biologische Vater, der niemals in seinem Leben in Erscheinung getreten ist. ${ }^{73}$ Ganz offensichtlich fehlt ihm dazu aber im gegenwärtigen Moment, da er bedingt durch pathologische Wahnvorstellungen seine mühsam aufgebaute Bibliothek verkauft hat, ${ }^{74} \mathrm{der}>$ ideologische Vater $<$, den es für eine sozioanalytische Durchdringung seines >Werdegangs $<$ und seiner aktuellen Lebenslage jedoch bräuchte:

»Als ich gerade von >bürgerlichen Existenzen< schrieb, dachte ich kurz über habituelle Differenzen nach und wollte unwillkürlich zu den >Feinen Unterschieden< von Bourdieu greifen, um drin zu lesen. Es war nur ein halber Gedanke, ein vorbewusster Wunsch. Er war nicht zu erfüllen. Denn wohin greifen? Da gibt es nichts mehr. Drei oder vier Bücher von Bourdieu hatten in meiner Bibliothek gestanden; alle sind sie weg. $\ll^{75}$

Bourdieu - so wird hier nahegelegt - ist ein notwendiger, in diesem Fall nicht (mehr und noch nicht wieder) vorhandener Ausgangs- und Bezugspunkt autosoziobiografischen Erzählens. Dass seine Bedeutung in dieser Weise, quasi ex negativo, betont wird, verdeutlicht aber auch, dass potenziell über die biografische, analytische und intertextuelle Verbindung zu Bourdieu eine genealogische Verwandtschaftsbeziehung unter den verschiedenen und vielfältigen Narrationen von der sozialen Herkunft gestiftet wird.

Zweitens ist $\mathrm{zu}$ beobachten, dass autosoziobiografische Texte sich auch unabhängig vom Bezugspunkt Bourdieu ganz grundlegend in ein größeres Geflecht von transclasse-Erzählungen einschreiben, das sie als eigenen Kosmos entwerfen. Dies

\footnotetext{
72 Vgl. auch: »Die Schule war meine Rettung gewesen. Genau dort setzt auch der gescheiterte Bildungsroman ein, der hier im ganzen Buch miterzählt wird.« (Melle [Anm. 69], 184); »Hier [am Jesuitenkolleg] hatte es angefangen, und hier wollte ich kurz hin.« (Melle [Anm. 69], 187).

73 An diese Feststellung ließe sich sicherlich noch eine umfassendere Erörterung der Genderkonstellationen autosoziobiografischer Darstellungen anschließen. So bedürfte etwa die Frage, inwiefern sich weibliche und männliche genealogische Linien in unterschiedlicher Weise für eine Verhandlung der Klassenproblematik eignen, einer vertiefenden Auseinandersetzung.

${ }^{74} \mathrm{Vgl}$. dazu: »Ich begann meine Bücher zu verkaufen. Thomas Mann musste als Erster daran glauben. Ich hatte ihn noch nie leiden können, fand seinen Stil unehrlich und parfümiert, und mit der vielgerühmten Ironie konnte ich schon gar nichts anfangen. [...] Weg mit den gesammelten Billigwerken von Goethe, Schiller, Mark Twain, die ich seit meiner Jugend mit mir herumschleppte. Weg mit Frisch, diesem senilen Identitätsstotterer und Erotomanen, weg mit Johnson, dem Alkoholchronisten, weg mit den Pfaffen Böll und Dürrenmatt. [...] Ich wollte die Bücher loswerden, den Ballast, der mich vermeintlich beschwerte. Die Geistesgeschichte sollte nur in meinem Geist weiterbestehen, und sie war eh zur Gespenstergeschichte geworden, zu einem Spuk, der mich verfolgte. Die Bücher waren alle kontaminiert von der Heilserwartung, die [...] in mir kulminierte.« (Melle [Anm. 69], 161 f.).

Zur Bedeutung des Bücherschranks als (bürgerlicher) Bildungsinstitution vgl. Aleida Assmann, »Der väterliche Bücherschrank. Über Vergangenheit und Zukunft der Bildung «, Zeitschrift für Pädagogik 50 (2004), 5-20. Im Gegensatz dazu vgl. zum Fernseher als Stigma des Proletarischen die aussagekräftige Darstellung von Christian Baron in Ein Mann seiner Klasse, Berlin 2020, 11.
}

75 Melle (Anm. 69), 215. 
tritt in aller Deutlichkeit bereits in Karin Strucks Klassenliebe von 1973 zutage, einem literarischen Kaleidoskop verschiedener transclasse- und Bildungsgeschichten. Das Buch stellt nicht nur den Versuch dar, das Leben der aus dem bäuerlichen und dem Arbeitermilieu stammenden Eltern und Großeltern festzuhalten. Vielmehr ist es eine wütende Auseinandersetzung der Ich-Erzählerin mit der Frage nach den Möglichkeiten und Unmöglichkeiten, Hoffnungen und Schwierigkeiten klassenübergreifender Liebesbeziehungen, aber auch klassenübergreifender Lern-/Lehrverhältnisse, und kann damit als eine frühe Autosoziobiografie eingestuft werden. Eine Lektüre von Klassenliebe sensibilisiert für den Umstand, dass deutschsprachige autobiografische Texte, die von der Erfahrung des Klassenwechsels berichten, bereits in den 1970er Jahren eine erste Konjunktur erlebten, ${ }^{76}$ wenn auch wahrscheinlich aus anderen Gründen als denjenigen, die den gegenwärtigen Erfolg dieses Genres bedingen. ${ }^{77}$ Dabei ist zu beobachten, dass eine Reihe der Texte, auf die Struck verweist, für aktuelle Autosoziobiografien und die von ihnen gestiftete Intertextualität nach wie vor eine zentrale Rolle spielen. ${ }^{78}$ Dies gilt etwa für Peter Handkes Wunschloses Unglück,

\footnotetext{
${ }^{76}$ Vgl. dazu Reinhold Grimm, »Elternspuren, Kindheitsmuster. Lebensdarstellung in der jüngsten deutschsprachigen Prosa «, in: Ders. u. Jost Hermand (Hrsg.), Vom Anderen zum Selbst. Beiträge zu Fragen der Biographie und Autobiographie, Königstein/Ts. 1982, 167-182 sowie Michaela Holdenried, »Die 70er Jahre: Everybody's Autobiography«, in: Dies., Autobiographie, Stuttgart 2000, 250-257.

77 Von der Nachkriegszeit bis in die Mitte der 1970er Jahre entstand in der BRD eine »nivellierte Mittelstandsgesellschaft « (Helmut Schelsky, 1965). Ende der 1960er und 1970er Jahren machte sich die damit verbundene Bildungsexpansion stark bemerkbar, die z.B. durch Einrichtungen wie das seit 1971 existierende Schüler- und Studierendenbafög befördert wurde. War einerseits sozialgeschichtlich damit ein >Aufstieg qua Bildung in einer bisher unbekannten Form möglich, so war die Gesellschaft der späten 1960er und 70er Jahre andererseits hochgradig für Klassenfragen sensibilisiert, während für die Zeit danach festzustellen ist, dass die Chancengerechtigkeit des Bildungssystems wieder abgebaut wurde und gleichzeitig die Kategorie der Klasse in der öffentlichen Diskussion kaum noch von Relevanz war bzw. die Rede von der Klassengesellschaft gar als verpönt galt. Inzwischen wird festgestellt, dass aktuell weniger eine >Wiederkehr der Klassengesellschaft zu beobachten ist, als vielmehr ein Bewusstsein dafür reaktiviert wird, dass Klassenstrukturen durchgängig, wenn auch in verschiedenen sozialen Ausprägungen eine zentrale Rolle für Prozesse gesellschaftlicher Hierarchiebildung gespielt haben und bis heute weiterhin massiv spielen. (Vgl. dazu Andreas Reckwitz, »Von der nivellierten Mittelstandsgesellschaft zur Drei-Klassen-Gesellschaft: Neue Mittelklasse, alte Mittelklasse, prekäre Klasse«, in: Ders., Das Ende der Illusion. Politik, Ökonomie und Kultur in der Spätmoderne, Berlin 2019, 63-133.).

Versucht man die autosoziobiografischen transclasse-Texte der 1970er Jahre und der Gegenwart zu diesen Entwicklungen in Bezug zu setzen, so kann man zu der Vermutung gelangen, dass Texte wie Strucks Klassenliebe von einer in den 1970er Jahren noch neuen Erfahrung des bildungsbedingten sozialen Aufstiegs und den damit verbundenen Schwierigkeiten (insbesondere für Frauen) erzählen, während das Narrativ von der Rückkehr zur sozialen Herkunft aktuell davon bestimmt wird, dass das Versprechen auf einen >Aufstieg qua Bildung « nicht mehr eingelöst wird und sich zugleich eine neue Gesellschaftsstruktur herausbildet, in der eine >neue Mittelklasse < sich über Bildungstitel und einen kulturellen Lebensstil, nicht aber unbedingt über ökonomischen Erfolg definiert, während die >alte Mittelschicht $<$ und die >Unterschicht $<$ an kulturellem Kapital einbüßen.

${ }^{78}$ Nicht unwichtig für die literaturhistorische und sozialgeschichtliche Einordnung dieses Befundes erscheint es, sich die Lebensdaten von Karin Struck, 1947-2006, in Erinnerung zu rufen. Struck wurde also sieben Jahre nach Annie Ernaux geboren, die bis heute für den autosoziobiografischen Diskurs entscheidende Impulse liefert und mit ihren Texten womöglich auch eine Art literarisches Gedächtnis oder Archiv an autosoziobiografischen Bezugstexten in der longue durée schafft, worauf auch jüngere Autor*innen zugreifen (können).
} 
Jack Londons Martin Eden oder auch für Texte von Georg Büchner, Albert Camus, Simone de Beauvoir oder Walt Whitman. ${ }^{79}$

Das von Struck verwendete intertextuelle Verfahren macht Ulrich Breuer sehr pointiert durch seine Einordnung von Klassenliebe als »Roman der Kommunikation « sichtbar:

»Dabei würde die Gattungsbezeichnung Roman auf das Konzept des autonomen, poetischen und absoluten Textes, das Moment der Kommunikation dagegen auf die angestrebte Demokratisierung des Schreibens verweisen. $\ll^{80}$

Diese Bestimmung lässt sich dahingehend erweitern, dass sich die kommunikativen Funktionen vor allem auf das Anliegen richten, der sozialen Isolation zu entkommen, geht es in Klassenliebe doch um den »Zusammenschluß derer, die einmal Arbeiter waren oder aus einer Arbeiterfamilie kommen. $\ll^{81}$ Dieses Vorhaben verbindet sich für sie mit der Notwendigkeit, eine >andere $<$ Schreibschule jenseits kanonischer Vorbilder finden zu müssen: »Wo kann ich in die Lehre gehen, schreiben zu lernen? $«^{82}$ Letztlich erfindet Struck für und in Klassenliebe eine Darstellungsweise, die von der intra- und intertextuellen Kommunikation mit anderen Texten lebt. ${ }^{83}$ Dafür wählt sie eben jene (zumeist nicht kanonisierten) Texte aus, die ebenfalls von einer Erfahrung des Klassenübergangs erzählen und auf dieser Grundlage gesellschaftliche Problemlagen in eine narrative Form kleiden. Damit wendet sie ein Verfahren an, das Bourdieu in Die biographische Illusion (1986) als Bruch mit der Tradition beschrieben hat, »das Leben als eine Geschichte « zu behandeln, »das heißt als kohärente Erzählung einer signifikanten und auf etwas zulaufenden Folge von Ereignissen $\ll .{ }^{84} \gg$ Darum $\ll$, so folgert Bourdieu, »ist es nur logisch, wenn man diejenigen um Beistand bittet, die mit dieser Tradition auf eben dem Terrain zu brechen

\footnotetext{
79 Andere wie beispielsweise Gerhard Zwerenz' Kopf und Bauch. Geschichte eines Arbeiters, der unter die Intellektuellen gefallen ist (1971) oder Hedwig Ortmanns Studie Arbeiterfamilie und sozialer Aufstieg. Kritik einer bildungspolitischen Leitvorstellung (1971) sind wohl weitgehend in Vergessenheit geraten und stellen für aktuelle Autosoziobiografien keine Referenzpunkte mehr dar.

${ }^{80}$ Ulrich Breuer, »Nackt wandern. Karin Strucks Klassenliebe im Bekenntnisdiskurs (der siebziger Jahre)«, Zeitschrift für Literaturwissenschaft und Linguistik 126 (2002) (Schwerpunkt: »Bekenntnisse«, hrsg. Wolfgang Haubrichs), 103-127, hier: 123.

81 Karin Struck, Klassenliebe. Ein Roman, Frankfurt a.M. 1973, 87.

82 Struck (Anm. 81), 270.

83 Erzählungen >aufsteigender< Arbeiterkinder werden in Klassenliebe gegen die Erzählungen der bürgerlichen Klasse abgegrenzt, so etwa in Auseinandersetzung mit »Paul Michael L.«: »Die Kette der Leiden... daß ich so darunter leide, zwischen zwei Klassen zu leben... Andere, siehe Simplicius, siehe aber auch Goethe und sogar Thomas Mann, hätten daraus ihr ganzes Überlegenheitsgefühl, ihren Humor und ihre Ironie geschöpft (alles stark simplifiziert). Jedenfalls müsse eine solche Lage nicht zu Depressionen führen. Das sehe er, Paul Michael L., ja bei sich selbst. Er würde jeden erschlagen, der ihn einen Bourgeois nenne, gleichzeitig wisse er genausogut, daß er nicht mehr zur Arbeiterklasse gehöre. Zu mehr Objektivität, Distanz und damit Urteilsvermögen sei eine solche >Zwischenlage< ganz gut. [...] Daß ich nicht lache: Goethe und Thomas Mann... Liest Paul Michael L. nicht Goethes Autobiographie... dieser Mensch, der die Bildung mit der Muttermilch einsaugt, und ich, was haben wir miteinander zu tun?« (Struck [Anm. 81], 86f. u. 90.) Eine ähnlich gelagerte Abgrenzung gegenüber der literarischen Hochkultur, nämlich von Kafkas Brief an den Vater und denjenigen, die sich auf diesen beziehen, findet sich in Bjerg (Anm. 62), 60. 84 Pierre Bourdieu, »Die biographische Illusion«, in: Ders., Praktische Vernunft. Zur Theorie des Handelns, Frankfurt a.M. 1998, 75-83, hier: 77 (Hervorhebung von mir, E.B.).
} 
hatten, auf dem sie zu ihrer exemplarischen Vollendung kam. $\ll^{85}$ Eben einen solchen Beistand sucht sich Struck. Dabei geht es in Klassenliebe um die Schaffung eines Kommunikationsraums ebenso wie um die deiktische Funktion einer nichtsingulären Erfahrung.

Ein ganz ähnlicher Befund steht bei Dröscher am Ende von Zeige deine Klasse, wenn sie feststellt:

»dass die Anwesenheit in seinem sozialen Raum - also der eigene Platz in der Gesellschaft - sehr unterschiedlich gestaltet werden kann. Was es voraussetzt, ist die Verweigerung von Einsamkeit (Spinoza). Es setzt voraus, sich zu zeigen, mit anderen zu sprechen. Sich mit-zu-teilen. $\ll^{86}$

Hier wird nochmals die Bedeutung von Allianzen betont und auf die kommunikativ-kollektivbildende Funktion von transclasse-Erzählungen hingewiesen, die eine Auseinandersetzung mit der eigenen Klassenherkunft in Form trans- und intragenerativer Narrationen zum Gegenstand haben. ${ }^{87}$ Für eine Klassengesellschaft in Transformation liegt aber gerade darin das gleichermaßen analytische wie politische Potenzial autosoziobiografischer Darstellungsverfahren.

\section{VI.}

\section{LITERATUR(WISSENSCHAFT) UND SOZIOLOGIE. EIN AUSBLICK}

Die hier diskutierte Problematisierung der Klassengesellschaft vollzieht sich trotz aller autobiografischer Bezüge im imaginären Raum von Film und Literatur. Das macht darauf aufmerksam, dass sich die Entstehung und Entwicklung von Kollektivitäten wie derjenigen der Klasse immer auch als Geschichte und Geschichten politisch relevanter Imaginationen erzählen lässt. ${ }^{88}$ Die autosoziobiografischen Darstellungen des Klassenwechsels verdeutlichen jedoch zudem, dass der Erwerb von Privilegien auch als Erfahrung eines Verlustes erlebt werden kann. Aus dieser Erfahrung heraus kann aber zugleich der Gewinn einer politischen Perspektivierung

\footnotetext{
85 Bourdieu (Anm. 84), 77.

86 Dröscher (Anm. 60), 244.

Bezeichnenderweise ist Spinoza auch für Jaquets Auseinandersetzung mit der Situation des transclasseIndividuums von entscheidender Bedeutung, entwickelt sie ihr Konzept der »complexion« doch ausgehend von dessen Begriff des »ingenium« (vgl. Jaquet [Anm. 63], 99).

87 Im Übrigen lässt sich auch Bourdieus Ein soziologischer Selbstversuch auf diese Weise lesen: Zeigt Bourdieu sich darin doch zunächst als ein in kollektiv-kommunikativen Zusammenhängen (des >Feldes der Akademia) stehendes Individuum (11-45), um dann von der Rückkehr in sein Herkunftsdorf in der Béarn zum Zwecke ethnografischer Forschungen über das Phänomen der Nicht-Verheiratung von Erstgeborenen (68-75) und schließlich erst danach seine soziale und familiäre Herkunftsgeschichte aus persönlicher Perspektive zu erzählen (95-102) - quasi als eine Form nachgetragener Ursprungsgeschichte zu seiner weiteren Entwicklung zum Soziologen mit einer spezifischen Sicht auf gesellschaftliche Phänomene (vgl. Pierre Bourdieu, Ein soziologischer Selbstversuch, Frankfurt a.M. 2002). Vgl. dazu auch im Folgenden unter Abschnitt VI.

$88 \mathrm{Vgl}$. dazu in grundlegender Perspektive Patrick Eiden-Offe, Die Poesie der Klasse. Romantischer Antikapitalismus und die Erfindung des Proletariats, Berlin 2017.
} 
resultieren, die in Gayatri Chakravorty Spivaks Forderung nach »unlearning one's privilege as one's loss $\ll^{89}$ prägnant zum Ausdruck kommt: Privilegien, die an eine bestimmte, oft bereits qua Geburt und Sozialisation verliehene, soziale Position gebunden sind, können den Blick auf Lebenserfahrungen und auf Formen der Bildung und des Wissens verstellen, die eben nicht mit diesem privilegierten Standpunkt einhergehen. Privilegien als Verlust einer Wahrnehmung marginalisierter Perspektiven im Sinne Spivaks aufzufassen, bedeutet, diesen eine besondere politische und gesellschaftliche Bedeutung zuzumessen und Aufmerksamkeit auf die Formen ihrer Artikulation zu richten. Genau dies leisten die diskutierten Beispiele autosoziobiografischer Filme und Texte. ${ }^{90}$

Die Vorstellung eines linearen Lebensverlaufs bzw. das Konzept einer festen Position wird zusätzlich grundlegend hinterfragt. Nicht nur stellen die Geschichten der Klassenübergänger*innen individuelle und soziale Mobilität aus und nutzen diese Erfahrung für einen >anderen $<$ Blick auf gesellschaftliche Dynamiken. ${ }^{91}$ Vielmehr imaginieren sie diese Geschichten, wie gezeigt wurde, in Form einer literarischen Kollektivierung, die mindestens zwei Strategien nutzt: Zum einen die Projektion individueller Lebensverläufe in das Geflecht intergenerativer Genealogi-

${ }^{89}$ Vgl. Donna Landry, Gerald MacLean, »Reading Spivak. Introduction«, in: Dies. (Hrsg.), The Spivak Reader. Selected Works of Gayatri Chakravorty Spivak, New York/London 1996, 4.

90 Vgl. für eine solche Sichtweise auch Wietschorke (Anm. 5).

Bei Bourdieu findet sich dazu dieser (lange und vielleicht nicht ganz zufällig recht opake) Satz: »Diese Art des Experimentierens mit der Arbeit der Reflexivität, die ich in meinen Untersuchungen über den Béarn und vor allem über das Verhältnis von Forschung und Forschendem praktiziert habe, zeigt [...], daß eine der kostbarsten Ressourcen jener praktischen Meisterschaft, die den Beruf des Soziologen auszeichnet und an der zuallererst das beteiligt scheint, was man Intuition nennt, am Ende vielleicht der wissenschaftliche Gebrauch einer gesellschaftlichen Erfahrung ist, die, wie sozial wertlos sie als solche auch immer sein mag und auch wenn sie von Krisen (der Konversion und Rekonversion) begleitet wird, sich unter der Voraussetzung ihrer kritischen soziologischen Reflexion von einem schweren Handicap in Kapital verwandeln kann.« (Bourdieu [Anm. 87], 74.).

Weiter gegen Ende von Ein soziologischer Selbstversuch führt Bourdieu dann Folgendes zur Bedeutung des/seines Klassenhintergrunds für die wissenschaftliche Praxis aus. Diese Ausführungen münden zudem in eine - auch für die vorliegenden Überlegungen - interessante Offenbarung: »Vielleicht ist [...] gerade die Tatsache, aus jenen >Klassen< zu kommen, deren Lebensverhältnisse man gerne >bescheiden $<$ nennt, für bestimmte Fähigkeiten verantwortlich, die in Handbüchern der Methodologie nicht gelehrt werden: das Fehlen einer jeden Geringschätzung für empirische Genauigkeit, eine Aufmerksamkeit für die scheinbar nebensächlichsten und >minderwertigsten`Untersuchungsgegenstände, der Widerwille gegen großartige Aufkündigungen des Bestehenden und aufsehenerregende geistige Glanzleistungen, ein Aristokratismus der Zurückhaltung, der in eine Mißachtung aller Erwartungen an jene intellektuelle Brillanz mündet, wie sie von den akademischen Institutionen und heute den Medien belohnt wird.

Und so habe ich mich auch, unter gänzlicher Vermeidung einer Rhetorik der Bedeutsamkeit, durch die sich der philosophische Dünkel so gerne ausspricht [...], immer wieder bemüht, meine theoretisch bedeutsamen Erkenntnisse in Einschüben oder Fußnoten zu verbannen, meine abstraktesten Überlegungen in hyperempirische Untersuchungen über solche Gegenstände einzubringen, die sozial nachrangig, politisch unbedeutend und intellektuell verpönt waren. Der erste Entwurf jeder späteren Theorie: Der Versuch etwa einer Überwindung der gegenseitigen Ausschließlichkeit von Objektivismus und Subjektivismus und der Rückgriff auf vermittelnde Konzepte wie das der Disposition, findet sich in einem kurzen Vorwort zu einem Gemeinschaftswerk über einen ziemlich abseitigen Gegenstand, den der Photographie." (Bourdieu [Anm. 87], 116f.; Hervorhebung von mir, E.B.).

91 Diese Lesart ist nicht kongruent zu derjenigen Spoerhases, der konstatiert: »Klassenübergänger [...] bleiben häufig von Identitätsvorstellungen besessen, die ein harmonisches und geschlossenes Selbst an die Stelle des hybriden Individuums setzen möchten.« (Spoerhase [Anm. 22], 252.). 
en von Familien und deren Klassenhintergründen und zum anderen die Herstellung eines intertextuellen Verbundes über Generationen von Schreibenden und ihre autosoziobiografischen Darstellungen hinweg. Nicht zuletzt verfolgen die Narrationen der Klassenwechsler*innen damit das - wenn auch oft in persönlich-individueller Hinsicht als illusorisch-utopisch markierte - Ziel, zumindest in Form der erzählenden Auseinandersetzung zu einer Wiederbegegnung mit der eigenen, inzwischen fremd gewordenen, Herkunftsklasse zu gelangen. So beschreibt bereits Bourdieu in Ein soziologischer Selbstversuch angesichts der Rückkehr in sein Heimatdorf zum Zwecke ethnografischer Studien

»das völlige Eintauchen in diese Umgebung und das Glück des Wiedersehens, von dem es begleitet wird, eine Aussöhnung mit den Dingen und den Menschen, von denen ich mich unmerklich entfernt hatte und die eine ethnografische Haltung ganz natürlich zu achten verpflichtet, die Jugendfreunde, die Eltern, ihr Verhalten, ihre Gewohnheiten, ihre Aussprache. Ein ganzer Teil meiner selbst wird mir wiedergegeben, jener, durch den ich ihnen ähnlich war und der mich ihnen gleichzeitig entfremdete, weil ich ihn nur verleugnen konnte, indem ich sie verleugnete, im Banne der Scham, die ich für sie und für mich empfand - die Rückkehr zu den Ursprüngen wird begleitet von einer dennoch beherrschten Rückkehr des Verdrängten. «"92

Bezeichnend ist, dass der Ausgangspunkt für diese Form der Rückkehr ein »Klassenbild (mein eigenes) ${ }^{93}$ ist. Die Unterhaltung mit einem ehemaligen Klassenkameraden über diese Fotografie führt zu einer Untersuchung über das Gebot der Ehelosigkeit für Erstgeborene im Béarn. ${ }^{94}$ Denn interessanterweise resultiert dieses Phänomen laut Bourdieu in einer umgekehrten sozialen Mobilität als der eigenen, nämlich in einer Deklassierung der Betroffenen: »Die Erstgeborenen der wohlhabenden Familien, einst eine gute >Partie <, wurden schlagartig in bloße Bauern verwandelt, in abstoßende und rohe Hinterwäldler [...].« ${ }^{95}$ Die Erfahrung des Klassenwechsels sensibilisiert für Prozesse sozialer Dynamiken und damit zusammenhängender Aufstiegs- und Abstiegsgeschichten - diese literaturwissenschaftlich und soziologisch zu lesen und auf ihr gesellschaftsdiagnostisches Potenzial hin zu befragen, kann angesichts der aktuellen Konjunktur von Autosoziobiografien als Aufforderung verstanden werden, die auch den Studien und Schriften Bourdieus eingeschrieben ist.

Dass im Raum von Literaturkritik und Wissenschaft im Moment vor allem diejenigen Erzählungen reüssieren, die das Narrativ der sozialen Herkunft aus der

\footnotetext{
92 Bourdieu (Anm. 87), 71.

93 Bourdieu (Anm. 87), 71.

94 Daneben ist für den erfolgreichen Fortgang und die wegweisende Erkenntnis von »Strategien« als Grund für die Heiratsregeln im Béarn »eine ganz alltägliche Bemerkung, ein Satz meiner Mutter« für Bourdieu zentral. Bezeichnenderweise verweigert Bourdieu eine genauere Explikation dieses Umstands: »Ich werde hier nicht versuchen, die tiefen Wandlungen dieser privilegierten Nähe zum Gegenstand verständlich zu machen, die einen Satz, der nur in einer >natürlichen Umgebungく, in einem alltäglichen Gespräch familiärer Vertrautheit fallen konnte, zu einem entscheidenden Hinweis werden ließ, der sich in ein ganzes Erklärungsmodell einfügte.« (Bourdieu [Anm. 87], 74.).
}

95 Bourdieu (Anm. 87), 73. 
Perspektive einer erworbenen sozialen Distanz und den damit einhergehenden Privilegien - auch dem Privileg, erzählen zu können und gehört zu werden - verfolgen, bedarf jedoch noch weiterer Erörterung, die auf eine kritische Hinterfragung der öffentlichen und wissenschaftlichen Rezeption abzielt. Denn die aktuelle Diskussion kreist unter anderem maßgeblich um die (von Bourdieu vernachlässigte) Frage, warum jemand den Klassenwechsel schafft und jemand anderes, die oder der aus den gleichen Verhältnissen stammt und womöglich dieselben Talente und Kapazitäten besitzt, nicht - ohne dabei, und darauf kommt es an, psychologisierende Erklärungen gelten zu lassen. ${ }^{96}$ Aus literaturwissenschaftlicher Sicht werden hingegen vor allem Fragen der Literarizität und Gattungszugehörigkeit autobiografischer Darstellungen weiter zu erörtern sein. So wird etwa vertiefend analysiert werden müssen, inwiefern sich die Darstellungsverfahren einer Schriftstellerin wie Annie Ernaux von denjenigen unterscheiden, die, wie bei Eribon, vom akademischen Beruf des Autors beeinflusst sind. Auch bedarf es eines Vergleichs von Texten, die dem Narrativ der Rückkehr zur sozialen Herkunft aus einer explizit autobiografischen Perspektive folgen, mit solchen Texten, die die Merkmale eines >autobiografischen Pakts $<$ nicht erfüllen, aber dennoch von der Rückkehr zur familiären, sozialen und geografischen Herkunft erzählen und ebenfalls als gegenwartsdiagnostisch aussagekräftige Texte gelesen werden. Texte wie Bjergs Roman Serpentinen, der bezeichnenderweise einen Soziologieprofessor zum Erzähler einer solchen Rückkehr zur Herkunft macht und den Bildungsaufstieg des Protagonisten als einen problematischen, wenn nicht gar als gescheiterten in Szene setzt, ${ }^{97}$ erscheinen dabei in mancherlei Hinsicht als kritische Kommentare und literarisch-fiktionale Infragestellungen des autosoziobiografischen Genres und seines Erfolgs. ${ }^{98}$

Insgesamt wird es im Zuge dieser Untersuchungen zu einer Aufweichung der »traditionelle[n] Arbeitsteilung zwischen einer textorientierten, hermeneutisch vorgehenden literaturwissenschaftlichen Perspektive und einer produktions- und rezeptionsorientierten soziologischen Fokussierung $^{99}$ kommen müssen. Wenn zu beobachten ist, dass soziologische Studien auf literarische Quellen zurückgreifen und zugleich Literaturwissenschaftler*innen das Verhältnis von Textform und soziopolitischem Aussagegehalt thematisieren, so steht eine engere Zusammenarbeit zwischen Soziologie und Literaturwissenschaft zumindest zur Diskussion, wobei die Ausrichtung der Untersuchungsperspektiven noch genauer zu bestimmen wäre. Das Verhältnis von Fiktion respektive Fiktionalität und gesellschaftlicher Realität/Gegenwart muss dabei erneut für eine transdisziplinäre Befragung geöffnet werden. Es steht zu vermuten, dass nicht zuletzt ein auch den wissenschaftlichen Disziplinen selbst

\footnotetext{
96 Vgl. dazu grundlegend Jaquet (Anm. 63), 29-102.

97 »Ich war jetzt Professor, und es hatte sich nichts verändert.« (Bjerg [Anm. 62], 113); »Ich erkannte die Forschung, die Theorien, natürlich, ich hatte Elias und Bourdieu gelesen und alle, die davor, danach und dazwischen kamen. Ich erkannte die Mechanik, doch dass diese Mechanik auch auf mich selbst wirkte, das war eine Erkenntnis, die privat bleiben musste.«(Bjerg [Anm. 62], 117.).

98 In diesem Zusammenhang lässt sich insbesondere auch Elena Ferrantes Die Geschichte des verlorenen Kindes (2014) anführen, in dem die Rezeption eines Romans der Ich-Erzählerin von Verlegern und Öffentlichkeit als autosoziobiografisch und daher in mehrfacher Hinsicht problematisch ausgestellt wird.

99 So formuliert ein Abstract zu einem am 07.02.2020 am KWI in Essen stattgefundenen Workshop zu »Literatur/Soziologie« unter der Leitung von Sina Farzin und Julika Griem.
} 
inhärenter Klassismus, der die Bewertung autosoziobiografischer Darstellungen und soziologischer Romane durch Literaturwissenschaft und Soziologie implizit bewegt, zutage treten könnte. Womöglich liegt in dessen Bearbeitung die eigentliche und größte wissenschaftshistorische und -soziologische Herausforderung.

Funding Open Access funding enabled and organized by Projekt DEAL.

Open Access Dieser Artikel wird unter der Creative Commons Namensnennung 4.0 International Lizenz veröffentlicht, welche die Nutzung, Vervielfältigung, Bearbeitung, Verbreitung und Wiedergabe in jeglichem Medium und Format erlaubt, sofern Sie den/die ursprünglichen Autor(en) und die Quelle ordnungsgemäß nennen, einen Link zur Creative Commons Lizenz beifügen und angeben, ob Änderungen vorgenommen wurden.

Die in diesem Artikel enthaltenen Bilder und sonstiges Drittmaterial unterliegen ebenfalls der genannten Creative Commons Lizenz, sofern sich aus der Abbildungslegende nichts anderes ergibt. Sofern das betreffende Material nicht unter der genannten Creative Commons Lizenz steht und die betreffende Handlung nicht nach gesetzlichen Vorschriften erlaubt ist, ist für die oben aufgeführten Weiterverwendungen des Materials die Einwilligung des jeweiligen Rechteinhabers einzuholen.

Weitere Details zur Lizenz entnehmen Sie bitte der Lizenzinformation auf http://creativecommons.org/ licenses/by/4.0/deed.de. 